\title{
Vitamin D and Its Synthetic Analogs
}

\author{
Miguel A. Maestro, ${ }^{\dagger}$ Ferdinand Molnár, ${ }^{\ddagger}$ and Carsten Carlberg*, ${ }^{\circledR}(0)$ \\ ${ }^{\dagger}$ Departamento de Química-CICA, Universidade da Coruña, ES-15071 A Coruña, Spain \\ ${ }^{\ddagger}$ School of Science and Technology, Department of Biology, Nazarbayev University, KZ-010000 Astana, Kazakhstan \\ ${ }^{\S}$ School of Medicine, Institute of Biomedicine, University of Eastern Finland, FI-70211 Kuopio, Finland
}

Supporting Information

\begin{abstract}
For many individuals, in particular during winter, supplementation with the secosteroid vitamin $\mathrm{D}_{3}$ is essential for the prevention of bone disorders, muscle weakness, autoimmune diseases, and possibly also different types of cancer. Vitamin $\mathrm{D}_{3}$ acts via its metabolite $1 \alpha, 25$ dihydroxyvitamin $\mathrm{D}_{3}\left[\mathbf{1 , 2 5}(\mathrm{OH})_{2} \mathrm{D}_{3}\right]$ as potent agonist of the transcription factor vitamin $\mathrm{D}$ receptor (VDR). Thus, vitamin $\mathrm{D}$ directly affects chromatin structure and gene regulation at thousands of genomic loci, i.e., the epigenome and transcriptome of its target tissues. Modifications of $1,25(\mathrm{OH})_{2} \mathrm{D}_{3}$ at its side-chain, A-ring, triene system, or C-ring, alone and in combination, as well as nonsteroidal mimics provided

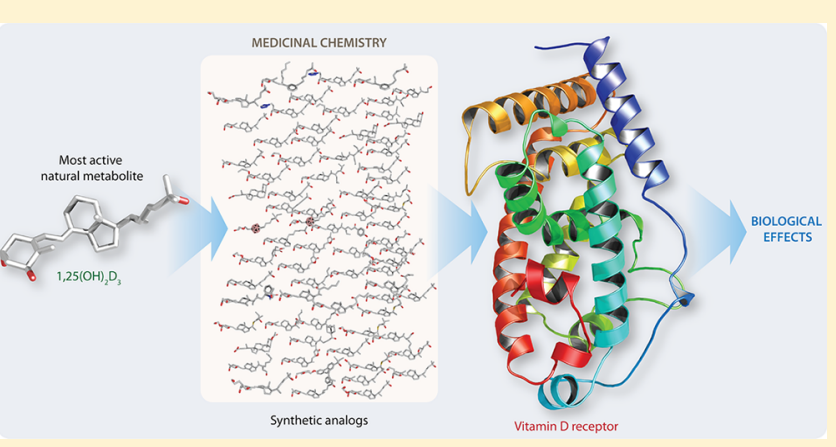
numerous potent VDR agonists and some antagonists. The nearly 150 crystal structures of VDR's ligand-binding domain with various vitamin $\mathrm{D}$ compounds allow a detailed molecular understanding of their action. This review discusses the most important vitamin $\mathrm{D}$ analogs presented during the past 10 years and molecular insight derived from new structural information on the VDR protein.
\end{abstract}

\section{INTRODUCTION}

An UV-B (290-315 nm)-dependent, nonenzymatic reaction in human skin converts the cholesterol precursor 7-dehydrocholesterol into previtamin $\mathrm{D}_{3}$ that further isomerizes into vitamin $\mathrm{D}_{3}$ (calciferol, 1$)^{1}$ (Figure 1). Similarly, UV-B-radiated plants and mushrooms are able to produce the isomer vitamin $\mathrm{D}_{2}$ (ergocalciferol, 2) based on their membrane sterol ergosterol. ${ }^{2}$ Both secosteroids are themselves biologically inert and have to be activated by hydroxylation first at C-25, leading to the prehormones 25-hydroxyvitamin $\mathrm{D}_{3}\left[\mathbf{2 5}(\mathbf{O H}) \mathrm{D}_{3}\right.$, (calcidiol, $3)]$ and $25(\mathrm{OH}) \mathrm{D}_{2}$, and then at C-1, creating $1,25(\mathrm{OH})_{2} \mathrm{D}_{3}$ (calcitriol, 4$)^{3}$ and $1,25(\mathrm{OH})_{2} \mathrm{D}_{2}$, respectively.

$\mathbf{2 5}(\mathrm{OH}) \mathrm{D}_{3}$ is the metabolically most stable and abundant vitamin $\mathrm{D}$ metabolite, and its serum levels serve as a biomarker of the vitamin $\mathrm{D}$ status of individuals. The biologically active form of vitamin $D_{3}, \mathbf{1}, 25(\mathrm{OH})_{2} \mathrm{D}_{3}$, acts via activation of the transcription factor VDR as a nuclear hormone that directly affects gene regulation. ${ }^{5}$ The physiological role of vitamin $\mathrm{D}$ is the regulation of calcium homeostasis for maintaining bone mineralization $^{6}$ as well as the modulation of innate and adaptive immunity ${ }^{7}$ for improving the response to infections by microbes, such as Mycobacterium tuberculosis, ${ }^{8}$ and preventing autoimmune diseases, such as multiple sclerosis. ${ }^{9}$

Lifestyle decisions, such as staying predominantly indoors and covered by textile outdoors, combined with changes in seasons and climate cause, for many individuals, insufficient exposure to UV-B and thus low endogenous production of vitamin $\mathrm{D}_{3}$. Human diet is often rather low in vitamin $\mathrm{D}$ because only fatty fish and UV-B irradiated mushrooms have reasonable quantities of the vitamin $D_{3}$ or vitamin $D_{2}$, respectively. The fortification of milk, margarine, and juices with vitamin $\mathrm{D}_{3}$ or vitamin $\mathrm{D}_{2}$ is applied in some countries. Moreover, in winter months daily supplementation with at least $25 \mu \mathrm{g}$ (1000 IU) of vitamin $\mathrm{D}_{3}$ is recommended in order to prevent vitamin D deficiency. ${ }^{10}$ The latter not only would result in rickets in children and in a higher risk of bone fractures due to osteoporosis or osteomalacia in adults, ${ }^{11}$ but also will compromise the function of the immune system and the claimed preventive actions of vitamin $\mathrm{D}$ against cardiovascular diseases, diabetes, neuropsychiatric disorders, and cancer. $^{12}$ Supplementation with vitamin $\mathrm{D}_{3}$ clearly increased in the general population, e.g., the sales of vitamin $\mathrm{D}$ supplementation products increased within 1 decade nearly 15-fold. ${ }^{13}$

There is no doubt that a sufficient vitamin $\mathrm{D}$ status is important for bone health, ${ }^{14}$ but overdosing with vitamin $\mathrm{D}_{3}$, $\mathbf{1 , 2 5}(\mathrm{OH})_{2} \mathrm{D}_{3}$, or its synthetic analogs may result in tissue calcification. ${ }^{15}$ Symptoms of hypercalcemia are (i) digestive distress, such as vomiting, nausea, and stomach pain, (ii) fatigue, dizziness, and confusion, (iii) excessive thirst, and (iv) frequent urination. However, hypercalcemia occurs rarely and no other severe side effects or toxicity of vitamin D overdosing is known. Nevertheless, higher doses of vitamin $D_{3}$ are not

Received: January 31, 2019

Published: March 27, 2019 


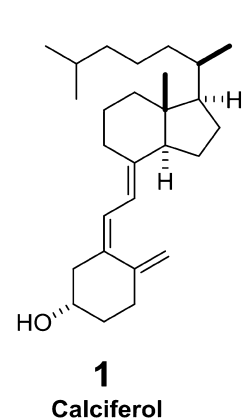

Calciferol<smiles>C=C1CC[C@H](O)C/C1=C/C=C1\CCCC23C/C(=C\C(C)C(C)C(C)C)C(C)[C@H]2CC[C@H]13</smiles>

2

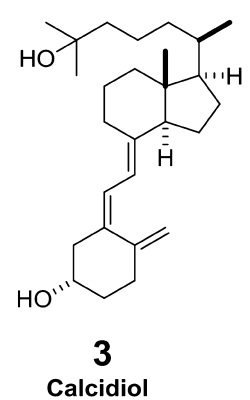

Calcidiol

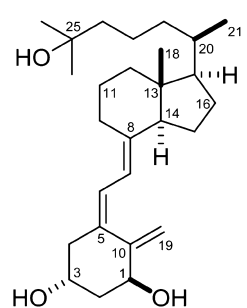

Calcitrio

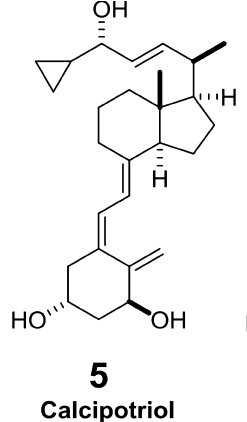<smiles>C=C1/C(=C\C=C2/CCCC34C=C(C(C)C(C)C(C)C)CC(C)C3CCC24)C[C@@H](O)CC1O</smiles>

Doxercalciferol<smiles>[3H]C1C[C@@H](O)C/C(=C/C=C2\CCCC3(CCCC(C)C)C2CCC3C(C)CCCC)C1=C</smiles>

Alfacalcidol

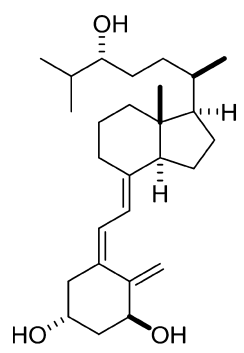

8

Tacalcitol<smiles>CC1C=CC23CCC/C(=C\C=C4CC(O)C[C@H](O)C4)C2CC[C@H]3C1C/C=C\C(C)C(C)(C)O</smiles>

9

Paricalcitol<smiles>C=C1/C(=C\C=C2/CCCC3(OCCC(C)(C)O)C(C)CCC23)C[C@@H](O)CC1O</smiles>

10<smiles>C=C1/C(=C\C=C2/CCCC3(CCCC(O)(F)C(F)(F)F)C(C)CCC23)C[C@@H](O)CC1O</smiles>

11

Oxacalcitriol<smiles>C=C1/C(=C\C=C2/CCCC3(CCCC(C)(C)O)CC4CC2C(C)CCC43)C[C@@H](O)C1OCCCO</smiles>

12

Eldecalcitol<smiles></smiles>

13

Inecalcitol

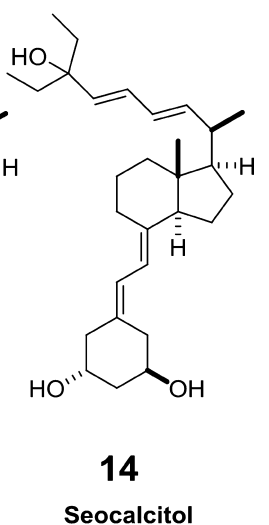

Figure 1. Vitamin D metabolites and analogs available on the market.

\section{Table 1. Vitamin D Compounds on the Market ${ }^{a}$}

- Vitamin $\mathrm{D}_{3}(\mathbf{1}$, calciferol) is used worldwide in the prevention of vitamin D deficiency and associated diseases, such as rickets [Vigantol (Bayer), Delsterol (DuPont), Duphafral- $\mathrm{D}_{3}$ (multiple pharma companies), Lutavit $\mathrm{D}_{3}$ (BASF), Vi-D3, Videkhol, Vigosan (multiple pharma companies)].

- Vitamin $\mathrm{D}_{2}$ (2, ergocalciferol) is also used in the prevention of vitamin $\mathrm{D}$ deficiency and associated diseases, such as rickets (marketed with different names by multiple pharma companies).

- Calcidiol $\left(3,25(\mathbf{O H}) \mathbf{D}_{3}\right)$ is used in the treatment of chronic hypocalcemia, renal osteodystrophy [Calderol (Upjohn), Hidroferol (Faes Farma)], rickets [Dedrogyl (Roussel), Hidroferol (Faes Farma)].

- Calcitriol $\left[4, \mathbf{1}, 25(\mathrm{OH})_{2} \mathrm{D}_{3}\right]$ is prescribed for renal osteodystrophy [Rocatrol (Roche), Calcijex (Abbott)], osteoporosis [Rocatrol (Roche)] and psoriasis [Silkis (Galderma)].

- Calcipotriol [5, 22-ene-26,27-dehydro-1,25(OH) $\left.{ }_{2} \mathrm{D}_{3}\right]$ is used for psoriasis [Davionex (Leo Pharmaceuticals), Dovonex (Warner Chilcott)].

- Doxercalciferol $\left[6,1 \alpha(\mathrm{OH}) \mathrm{D}_{2}\right.$, Hectorol (Bone Care International)] is prescribed for secondary hyperparathyrodism.

- Alfacalcidol $\left(7,1 \alpha(\mathrm{OH}) \mathrm{D}_{3}\right)$ is used for renal osteodystrophy [Alfarol (Chugai Pharmaceutical), One-Alpha (Leo Pharmaceuticals)], secondary hyperparathyrodism [Alfarol (Chugai Pharmaceutical)], osteoporosis [Alfarol (Chugai Pharmaceutical), Alpha $\mathrm{D}_{3}($ Teva Pharmaceuticals)] and rickets [Alfarol (Chugai Pharmaceutical)].

- Tacalcitol $\left(8,1 \alpha, 24(\mathrm{OH})_{2} \mathrm{D}_{3}\right)$ is prescribed for psoriasis [Bonalfa (Teijin), Curatoderm (Merck KGaA)].

- Paricalcitol [9, 19-nor-1,25(OH $)_{2} \mathrm{D}_{2}$, Zemplar, (Abbott Laboratories)] is used for secondary hyperparathyrodism.

- Oxacalcitriol $\left.(10,22 \text {-oxa-1,25(OH) })_{2} \mathrm{D}_{3}\right)$ is used for secondary hyperparathyrodism and psoriasis [Oxarol (Chugai Pharmaceuticals)] in Japan.

- Falecalcitriol $\left[11,1,25(\mathrm{OH})_{2}-26,27-\mathrm{F}_{6}-\mathrm{D}_{3}\right]$ is prescribed for secondary hyper-parathyrodism in Japan [Hornel (Taisho Pharmaceuticals and Sumitomo Pharmaceuticals), Fulstan (Kissei Pharmaceuticals)].

- Eldecalcitol [12, 2 $\alpha$-(3-hydroxypropoxy)-1,25(OH) ${ }_{2} \mathrm{D}_{3}$ ] is prescribed for osteoporosis only in Japan [Edirol (Chugai Pharmaceutical)].

${ }^{a}$ Only a few vitamin D compounds have reached the market. ${ }^{88,89}$ Their applications, commercial name, and company are listed. The structures of the compounds are shown in Figure 1.

recommended as nutritional supplement for reaching nonskeletal effects of the vitamin. Similarly, the main goal of the development of vitamin $\mathrm{D}$ analogs is to identify compounds with a low calcemic effect versus a potent antiproliferative, prodifferentiating, and/or immune-modulatory function.

In total, more than 3000 synthetic vitamin D analogs were developed by various pharmaceutical companies and academic research groups in order to advance the biological properties of the natural compound for a applications in the therapy of (i) hyperproliferative diseases, such as different types of cancer, (ii) psoriasis, an autoimmune disease of the skin, ${ }^{16}$ or (iii) bone disorders, such as osteoporosis. ${ }^{17}$ However, so far only a few vitamin $\mathrm{D}$ compounds made it to the market (Table 1 ). In addition to vitamin $\mathrm{D}_{3}$ being extensively used as a nutritional supplement, the commercially most successful vitamin D analog is calcipotriol (5), which is topical agent in clinical use for the treatment of psoriasis. Together with the compounds doxercalciferol (6), alfacalcidol (7), tacalcitol (8), paricalcitol (9), oxacalcitriol (10), falecalcitriol (11), and eldecalcitol (12) it had been discussed in previous reviews ${ }^{18,19}$ (Figure 1). In contrast, despite promising in vitro results, analogs such as inecalcitol (13) or seocalcitol (14) were unsuccessful in phase II clinical trials of acute myeloid leukemia (www.hybrigenics. $\mathrm{com} /$ news/articles/list/type/2) or pancreatic cancer, ${ }^{20}$ respectively. Interestingly, some immune-system-related vitamin D target genes, such as cathelicidin antimicrobial peptide 

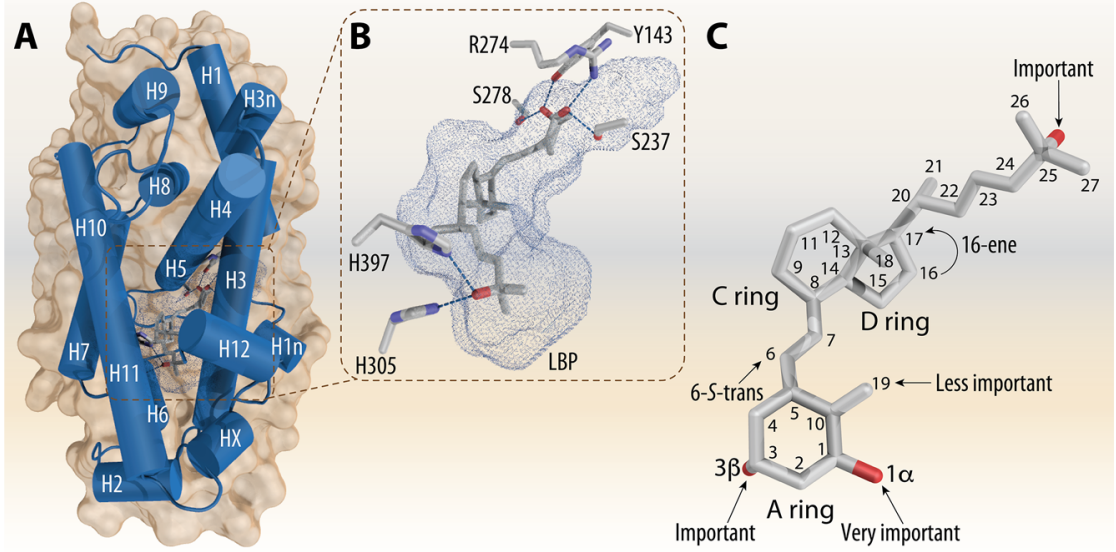

Figure 2. 1,25 $(\mathrm{OH})_{2} \mathrm{D}_{3}$ complexed to the VDR-LBD. The VDR-LBD has a conserved 3D architecture, which is made of a three-layer $\alpha$-helical sandwich. In the lower part of the LBD the LBP is located. All the helices are labeled from N-terminus toward C-terminus and numbered in white color (A). Details on the LBP with bound $1,25(\mathrm{OH})_{2} \mathrm{D}_{3}$ and critical amino acids that provide anchoring contacts for the three OH groups (B). Details on the conformation of the bound $1,25(\mathrm{OH})_{2} \mathrm{D}_{3}$ molecule with the annotated $\mathrm{OH}$ groups and highlights to its contribution of its activity. The numbering of the carbons atoms is indicated (C). The figure is based on the PDB code 1DB1.

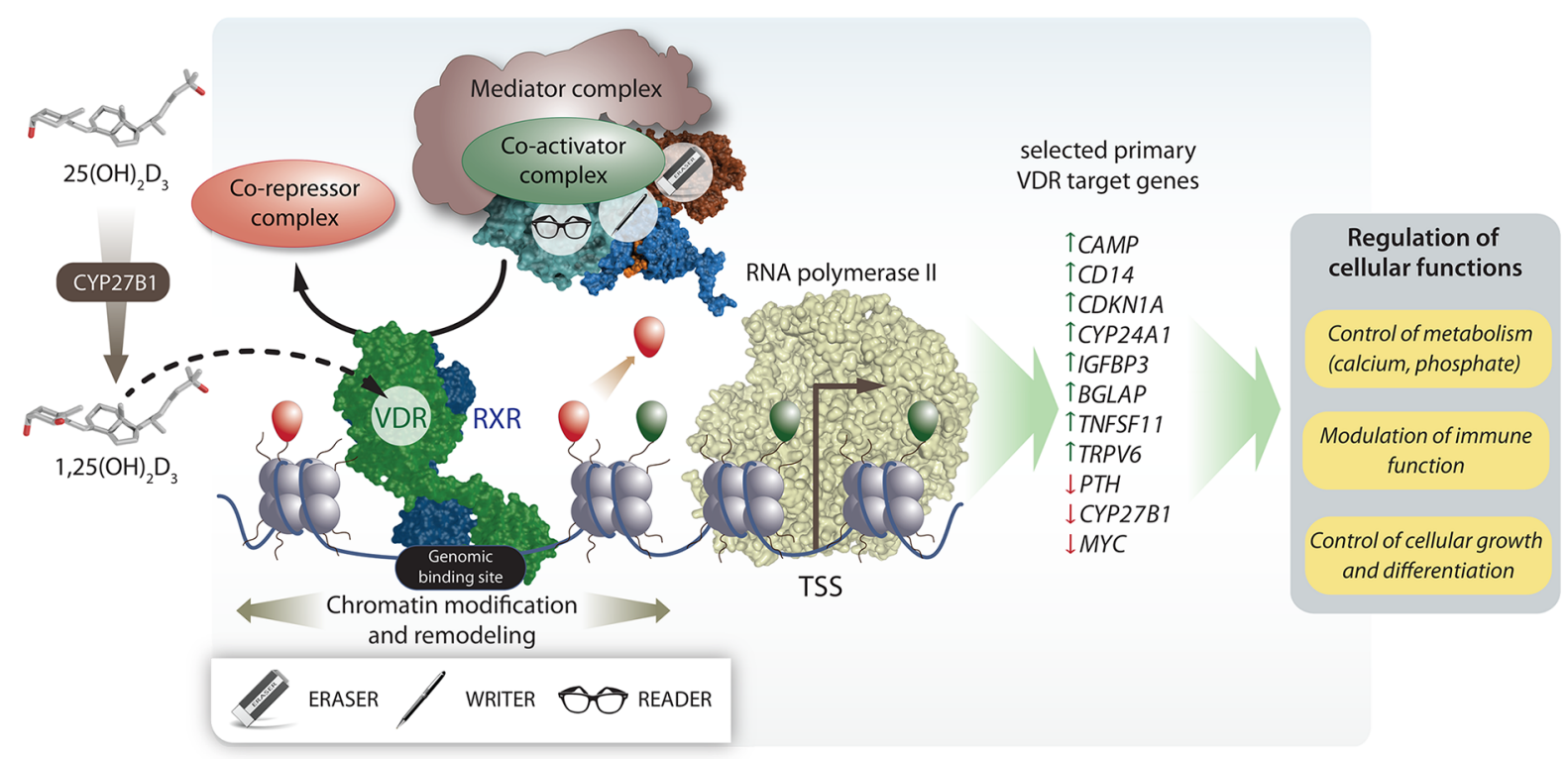

Figure 3. Vitamin D signaling. $25(\mathrm{OH}) \mathrm{D}_{3}$ is converted by the enzyme CYP27B1 to its biologically most active form $1,25(\mathrm{OH})_{2} \mathrm{D}_{3}$, which binds to the transcription factor VDR. Upon binding of $1,25(\mathrm{OH})_{2} \mathrm{D}_{3}$ or synthetic agonists, a conformational change in the LBD is induced leading to cofactor exchanges shifting the balance toward recruitment of coactivator proteins. Co-repressor proteins dissociate from the VDR-RXR heterodimer. In parallel, the mediator complex and chromatin modifying enzymes (readers, writers, and erasers) are recruited in order to handle histone proteins of local nucleosomes around genomic VDR binding sites. In addition, chromatin remodeling complexes are recruited and rearrange nucleosomes at vitamin $\mathrm{D}$-sensitive chromatin regions. Altogether, these chances lead to looping of the distal regulatory elements toward the basal transcriptional machinery with RNA polymerase II and other nuclear adaptor proteins initiating the start of $\mathbf{1 , 2 5}(\mathbf{O H})_{2} \mathbf{D}_{3}$-dependent transcription from hundreds to thousands of TSS regions throughout the whole human genome. The ultimate outcome is the increase or decrease of the of primary vitamin D target gene expression followed by changes of indicated cellular functions.

$(C A M P)$ and CD14 (encoding for a Toll-like receptor 4 coreceptor), are very responsive, ${ }^{21,22}$ while there are no vitamin $\mathrm{D}$ target genes with comparable inducibility involved in the management of cellular growth and differentiation. The failure of anticancer trials and the success in the therapy of an immune disease as well as prominent gene regulatory effects in immune cells suggest that VDR ligands, in addition to bonerelated functions, may rather have a therapeutic potential in immune diseases than in cancer.
The majority of synthetic VDR ligands are direct derivatives of $1,25(\mathrm{OH})_{2} \mathrm{D}_{3}$, but within the past years an increasing number of vitamin $\mathrm{D}$ mimics were published. $1,25(\mathrm{OH})_{2} \mathrm{D}_{3}$ had been modified at its side-chain, A-ring (often together with side-chain changes), triene system, and C-ring. These modifications follow the strategy to increase the VDR binding affinity while in parallel modulating the metabolic stability of the molecules. ${ }^{18}$ A reasonable number of new vitamin $D$ analogs have been published within the past years and will be 
<smiles>C=C1/C(=C\C=C2/CCC[C@@]3(C)[C@H](C#CC#CC(C)(C)O)CC[C@@H]23)C[C@H](O)CC1O</smiles>

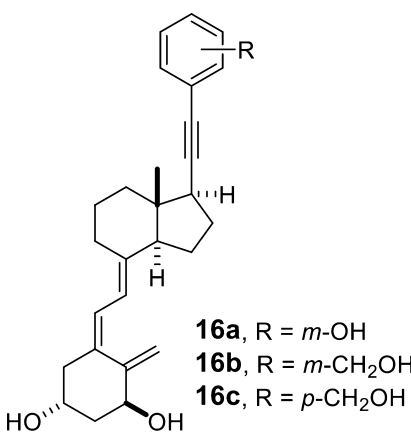<smiles>C=C1C(=CC=C2C[C@@H](O)CC(O)C2=C)CCCC1=CC=C1CCCC2(C)[C@@H]1CC[C@]2(C)C#CC#CC(C)(C)O</smiles>

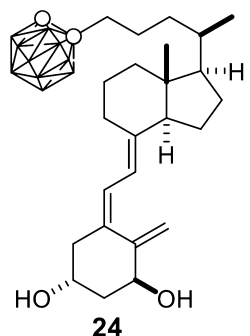<smiles>[R]C1CCC2C=CCCCC2(C)C1</smiles>

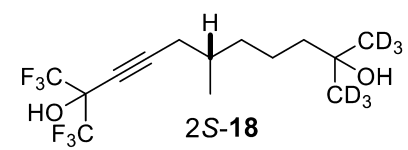

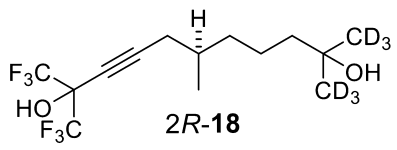<smiles>CC(CC=CC(C)(C)C(F)(F)F)CCCC(C)(C)C(=O)O</smiles>
$\mathrm{HO}$

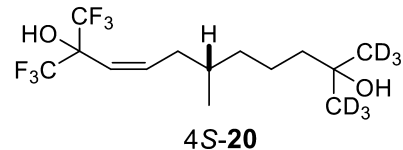

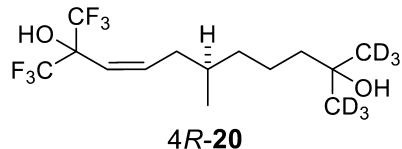<smiles></smiles><smiles>[R]C1CCC2C=CCCCC2(C)C1C</smiles>

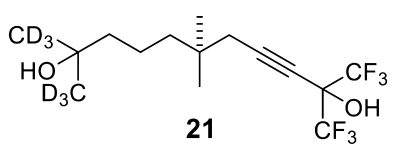

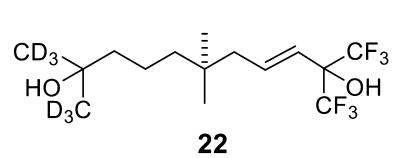<smiles>CC(C)([18O])CCCC(C)(C)CCC(C)(C)C(=O)O</smiles>

\begin{tabular}{|c|c|c|c|c|c|}
\hline Family & compound & VDR aff. & Prolif inh. & Transac act. & Calcemia \\
\hline \multirow{6}{*}{ 20(22)-yn- } & 15 & + & & & \\
\hline & $16 a$ & $=$ & & & \\
\hline & $16 \mathrm{~b}$ & $=$ & & & \\
\hline & $16 \mathrm{c}$ & - & & & \\
\hline & $17 a$ & ++ & & & \\
\hline & $17 \mathrm{~b}$ & ++ & & & \\
\hline \multirow{9}{*}{ Gemini } & $2 S-18$ & +++ & & & \\
\hline & $2 R-18$ & +++ & & & \\
\hline & $2 S-19$ & ++ & & & \\
\hline & $2 R-19$ & +++ & & & \\
\hline & $2 S-20$ & + & & & \\
\hline & $2 R-20$ & ++ & & & \\
\hline & 21 & & +++ & & \\
\hline & 22 & & +++ & & \\
\hline & 23 & & +++ & & \\
\hline Carboranyl & 24 & ++ & ++ & ++ & - \\
\hline
\end{tabular}

Ref: $1,25(\mathrm{OH})_{2} \mathrm{D}_{3}$ :

Figure 4. Side-chain-modified vitamin $\mathrm{D}$ analogs. The table summarizes the biological properties of the compounds: reference, $\mathbf{1 , 2 5}(\mathbf{O H})_{2} \mathrm{D}_{3} ;(=)$ similar value; $(+)>10 \times$ higher; $(++)>100 \times$ higher; $(+++)>1000 \times$ higher; $(-)>10 \times$ lower; VDR aff, VDR affinity; Prolif inh, proliferation inhibition; Transac act, VDR transactivation activity; calcemia, $\left[\mathrm{Ca}^{2+}\right]$ level changes in serum.

discussed in this review. Moreover, the number of solved VDR crystal structures with synthetic ligands has significantly grown.

\section{CENTRAL ROLE OF VDR IN VITAMIN D SIGNALING}

VDR is the only protein expressed by the human genome that is able to bind $1,25(\mathrm{OH})_{2} \mathrm{D}_{3}$ and its analogs at subnanomolar concentrations. ${ }^{23}$ Thus, all physiological functions of vitamin D compounds are mediated by VDR and its target genes. ${ }^{24}$ The $V D R$ gene is expressed most prominently in intestine, kidneys, and bone, but in most of the other 400 human tissues and cell types some VDR expression is found. ${ }^{25}$ This means that not only tissues that relate to calcium homeostasis and bone formation but also immune cells respond to vitamin $\mathrm{D} .^{26}$

VDR is an endocrine receptor and member of the superfamily of nuclear receptors; i.e., the mechanisms of its action are comparable to the receptors for glucocorticoids and estrogen. ${ }^{27}$ VDR's ligand-binding domain (LBD) is structurally conserved and comprises 11-15 $\alpha$-helices, modestly varies 
between solved crystal complexes, and depends on the folding of the intrinsically disordered region between helices $\mathrm{H} 1$ and $\mathrm{H} 3$ and the presence of a helix HX between helices H11 and $\mathrm{H}_{12}{ }^{28,29}$ (Figure 2A). The lower part of the LBD contains a ligand-binding pocket (LBP), which is a cavity with a volume of $\sim 700 \AA^{3}$ (with possible expansion beyond $1000 \AA^{3}$ ) being formed by some 40 mostly nonpolar amino acids. ${ }^{30}$ Three pairs of polar amino acids within the LBP fix via hydrogen bonds each one of the three $\mathrm{OH}$ groups (at $\mathrm{C}-1 \alpha, \mathrm{C}-3 \beta$, and $\mathrm{C}-25)$ of $1,25(\mathrm{OH})_{2} \mathrm{D}_{3}$. The $1 \alpha-\mathrm{OH}$ group interacts with Y143 (helix H1) and S278 (helix H5), the $3 \beta-\mathrm{OH}$ group contacts S237 (helix H3) and R274 (helix H5), and the 25$\mathrm{OH}$ group interferes with $\mathrm{H} 305$ (loop between helices $\mathrm{H} 6$ and H7) and H397 (helix H11) ${ }^{28}$ (Figure 2B).

VDR ligands induce a conformational shift to the LBD, which replaces co-repressor proteins by coactivator proteins; i.e., ligand binding induces a different protein-protein interaction profile of the receptor. ${ }^{31}$ VDR agonists cause an efficient dissociation of co-repressors from the LBD and allow the specific binding of coactivators and the mediator complex (Figure 3). Coactivators also attract chromatin modifying enzymes that write, erase, or read post-translational marks of histones, such as acetyl and methyl groups, to histone proteins of nucleosomes in the vicinity of genomic VDR binding sites. ${ }^{32}$ Moreover, also members of chromatin remodeling complexes interact in a ligand-dependent fashion with VDR and cause a rearrangement of nucleosomes at vitamin $\mathrm{D}$-sensitive chromatin regions. ${ }^{33}$ These epigenetic changes allow looping of VDRbound enhancers toward accessible transcription start sites (TSSs) at hundreds to thousands of loci throughout the human genome. ${ }^{34}$ These enhancer-TSS assemblies are triggered by ligand-activated VDR and finally result in an increase or decrease in the expression of hundreds of primary vitamin $\mathrm{D}$ target genes (Figure 3 ).

The structure of the human VDR-LBD complexed with $1,25(\mathrm{OH})_{2} \mathrm{D}_{3}$ was solved in the year $2000 .^{28}$ Since then altogether 143 human, rat, and zebrafish VDR-LBDs have been crystallized with a large number of synthetic analogs ${ }^{35}$ (Table $\mathrm{S} 1)$. In general, the analogs behave like $\mathbf{1 , 2 5}(\mathrm{OH})_{2} \mathrm{D}_{3}$ by stabilizing the $\mathrm{LBD}$ in more or less the same conformation, since the three $\mathrm{OH}$ groups of each vitamin $\mathrm{D}$ compound take up a nearly identical position. This suggests that there is only one agonistic conformation of the LBD for which the interaction between the ligand's $25-\mathrm{OH}$ group and the LBP amino acids $\mathrm{H} 305$ and $\mathrm{H} 397$ are most important (Figure 2C).

On the basis of the vitamin D analog's chemical modification, all solved VDR-ligand-complexes can be divided to the six groups: (i) A-ring modifications, (ii) side-chain modifications, (iii) triene system modifications, (iv) combined A-ring and side-chain modification, (v) modifications in the CD-ring, and (vi) nonsteroidal analogs. All modifications aim to either (i) maintain the three anchoring $\mathrm{OH}$ groups at the same position as in $\mathbf{1 , 2 5}(\mathrm{OH})_{2} \mathrm{D}_{3}$ and/or (ii) fill the LBP most efficiently in order to form additional hydrogen network and/ or hydrophobic contacts. More variant modifications of $1,25(\mathrm{OH})_{2} \mathrm{D}_{3}$ aim to alter the ligand conformation or to bounce the shape of the LBP by adding an additional sidechain at positions C-20 or C-22. Moreover, de novo designed nonsteroidal compounds carry modifications, such as the exchange the classical secosteroid ring structure by rings with aromatic character. The aim with these molecules is to maintain the hydrophobic interactions with amino acid residues lining the inner surface of the LBP as well as to increase the stacking interaction with aromatic amino acid residues.

In this review we discuss different classes of vitamin D analogs and, where applicable, provide molecular understanding from VDR crystal structures.

\section{SIDE-CHAIN MODIFICATIONS}

The first locked side-chain vitamin $\mathrm{D}$ analogs nor-21$20(22), 23(24)$ - diyn-1,25( OH $)_{2} \mathrm{D}_{3}$ (15), nor 21,23,24,25,26,27-20(22)-yn-22-(3-hydroxyphenyl)-1,25$(\mathrm{OH})_{2} \mathrm{D}_{3}$ (16a), nor-21,23,24,25,26,27-20(22)-yn-22-[3(hydroxymethyl)phenyl]-1,25( $\mathrm{OH})_{2} \mathrm{D}_{3}(\mathbf{1 6 b})$, and nor21,23,24,25,26,27-20(22)-yn-22-[4-(hydroxymethyl)phenyl]$1,25(\mathrm{OH})_{2} \mathrm{D}_{3}(16 \mathrm{c})$ have been synthesized by convergent route through a Wittig-Horner approach starting from Inhoffen-Lythgoe diol $^{36}$ (Figure 4). These analogs lead to significant activation of VDR-dependent transcription in comparison to $1,25(\mathrm{OH})_{2} \mathrm{D}_{3}$. An unique structural modification on the C-22-diyne analog, a C-17-methyl substitution, was provided through a vinyl(pinacolo)boronate approach and resulted in the C-17-methyl-substituted vitamin $\mathrm{D}$ analogs nor21-20(22),23(24)-diyn-17-methyl-1,25( $\mathrm{OH})_{2} \mathrm{D}_{3} \quad(17 \mathrm{a})$ and nor-21-20(22),23(24)-diyn-17-methyl-26,26,26,27,27,27-hexafluoro-1,25( $\mathrm{OH})_{2} \mathrm{D}_{3}(\mathbf{1 7 b}) .{ }^{37}$ The C-22-aromatic-substituted analogs are less potent in activating VDR than the C-22-diyne isomers. The C-17-methyl analogs bind more efficiently to VDR than $1,25(\mathrm{OH})_{2} \mathrm{D}_{3}$.

The two side-chain analog Gemini comprises an unaltered side-chain of $1,25(\mathbf{O H})_{2} \mathbf{D}_{3}$ and a second chain at C-20. ${ }^{38,39}$ Although the volume of Gemini is increased by some $25 \%$, it still fits into VDR's LBP. ${ }^{30}$ One side-chain of Gemini takes the same place as that of $\mathbf{1 , 2 5}(\mathrm{OH})_{2} \mathrm{D}_{3}$, whereas an extra subcavity opens within the LBP for the second side-chain. ${ }^{40}$ The increase in transcriptional activity of $\mathrm{Gemini}^{41}$ motivated the preparation of Gemini-type analogs with side-chains containing double or triple bonds and isohexafluoro-2-propanol or isohexadeutero-2-propanol side-chain ends. Compounds 1820 have been synthesized with both configurations at C-20 by a convergent approach through Wittig-Horner coupling starting from Inhoffen-Lythgoe diol. ${ }^{42}(R)$-Analogs showed higher antiproliferative potency in MCF10CA1 human breast cancer cells than their $(S)$-counterparts, and both were 1001000 times more potent than $1,25(\mathbf{O H})_{2} \mathbf{D}_{3}$. Furthermore, both configurations of the Gemini derivatives are also more potent than $1,25(\mathrm{OH})_{2} \mathrm{D}_{3}$ in inducing the differentiation of NB4 human leukemia cells. Thus, Gemini compounds have enhanced potency in inhibiting proliferation and inducing differentiation with reduced induction of hypercalcemia when compared to $1,25(\mathrm{OH})_{2} \mathrm{D}_{3}$. Moreover, C-20 methyl-substituted Gemini analogs $(\mathbf{2 1 - 2 3})$ are also potent in the inhibition of HL-60 human leukemia cell proliferation and the induction of CAMP gene expression. ${ }^{43}$

$1 \alpha$-Hydroxy-25,26,27-trinor-24-o-carboranyl-vitamin $\mathrm{D}_{3}$ $\left(1,25 \mathrm{cD}_{3}, \mathbf{2 4}\right.$, Figure 4$)$ is a rather new vitamin $\mathrm{D}$ analog, in which an $o$-carborane moiety replaces the $25-\mathrm{OH}$ group. ${ }^{44}$

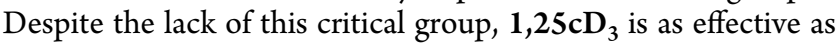
$\mathbf{1 , 2 5}(\mathrm{OH})_{2} \mathrm{D}_{3}$ in inhibiting the growth of MCF-7 human breast cancer cells and in inducing the differentiation of $\mathrm{HaCaT}$ human keratinocytes. VDR binds $1,25 \mathrm{cD}_{3} 2$ times tighter than $1,25(\mathrm{OH})_{2} \mathrm{D}_{3}$ and is equally potent as the natural hormone in inducing reporter gene activity while not showing adverse calcemic effects. Moreover, like most other vitamin D analogs, the conformation of the complex of $1,25 \mathrm{cD}_{3}$ with 


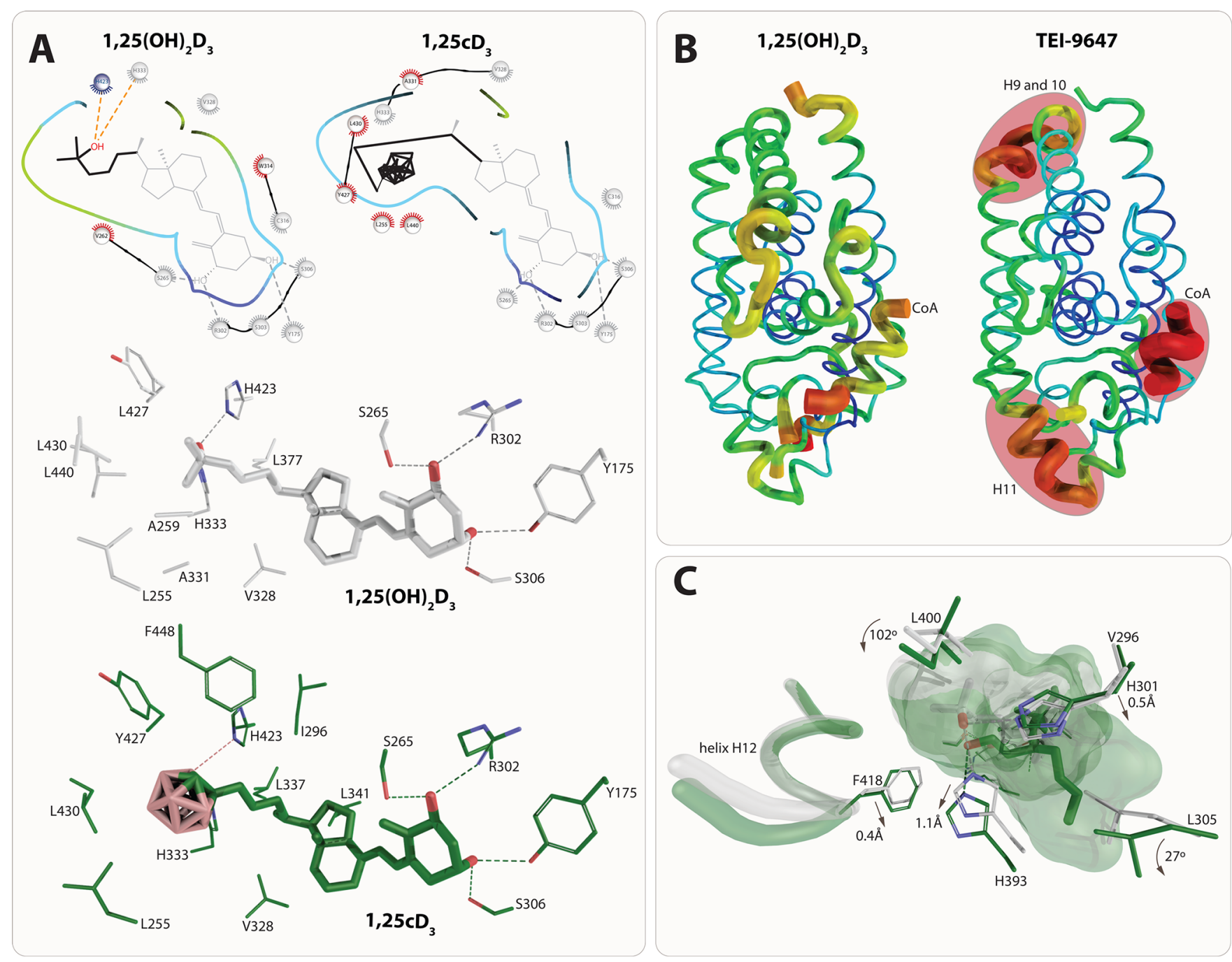

Figure 5. Structure-function relationship of VDR ligands (I). The carborane group of $\mathbf{1 , 2 5} \mathbf{c D}_{3}$ creates additional hydrophobic interactions that compensate for the loss of the $25-\mathrm{OH}$ group. All residues that have conserved interactions are shown in gray (top). Detailed interaction with residues based on PDB code structures $2 \mathrm{HC} 4\left(\mathbf{1 , 2 5}(\mathrm{OH})_{2} \mathrm{D}_{3}\right)$ and $5 \mathrm{E} 7 \mathrm{~V}\left(\mathbf{1 , 2 5} \mathrm{cD}_{3}\right)$. The displayed interactions are identified under cutoff $3.5 \AA$ (A). Destabilization of the VDR-LBD upon binding of 23,36-lactone analogs. Representation of crystal structure $b$-factors using structures PDB codes 1 RK3 $\left(\mathbf{1}, 25(\mathrm{OH})_{2} \mathbf{D}_{3}\right.$, left) and 3A2H (TEI-9647, right). Regions with the highest $b$-factors are highlighted in red and they are helices H910 that may affect heterodimerization with RXR, helix $\mathrm{H} 11$ affecting the position of helix H12, and the coactivator peptide showing very high $b$ factors overall. The most stabile part of the VDR is shown in blue through green, yellow and red monitoring the highest $b$-factor values (B). Structural implication of 22S-alkyl-2-methylene-19-nor-1,25 $(\mathrm{OH})_{2} \mathrm{D}_{3}$ binding. The position of the helix $\mathrm{H} 12$ takes the same conformation in both 22S-alkyl-2-methylene-19-nor-1,25( $\mathrm{OH})_{2} \mathrm{D}_{3}$ and $\mathbf{1 , 2 5}(\mathrm{OH})_{2} \mathrm{D}_{3}$. Many residues around the two aliphatic chains move or rotate, such as $\mathrm{H} 301$ (loop helices H6/7), H393 (helix H11), or F418 (helix H12). Structural elements from 22S-alkyl-2-methylene-19-nor-1,25(OH) ${ }_{2} \mathrm{D}_{3}(\mathrm{PDB}$ code 2ZXM) and $\mathbf{1 , 2 5}(\mathrm{OH})_{2} \mathrm{D}_{3}$ (PDB code $1 \mathrm{RK} 3$ ) complexes are highlighted in green and white color, respectively $(\mathrm{C})$.

VDR's LBD is highly similar to that of $1,25(\mathrm{OH})_{2} \mathrm{D}_{3}$; i.e. the protein shows the same topology. Nevertheless, the loop between helices $\mathrm{H} 6$ and $\mathrm{H} 7$, which is a critical region for the activation of the receptor, and the last part of helix H11 show shifts by $0.6 \AA$. The carborane side-chain is $2.4 \AA$ longer than that of $1,25(\mathrm{OH})_{2} \mathrm{D}_{3}$, but it is hydrophobic and therefore favors the interaction with hydrophobic amino acid in this part of the VDR-LBP. This compensates for the loss of the 25-OH group (Figure 5A). Thus, the collection of small changes stabilizes helices $\mathrm{H} 3, \mathrm{H} 11$, and $\mathrm{H} 12$ and overall causes higher stability of VDR's LBD.

\section{A-RING MODIFICATION}

The biological profiles of the C-2-substituted vitamin D analogs 2-(3'-hydroxypropyl)-1,25( $\mathrm{OH})_{2} \mathrm{D}_{3} \quad$ (25), 2-butyl-
$1,25(\mathrm{OH})_{2} \mathrm{D}_{3}$ (26), 2-(4'-hydroxybutyl)-1,25( OH $)_{2} \mathrm{D}_{3}$ (27), 2-(2'-hydroxyethoxy)-1,25( $\mathrm{OH})_{2} \mathrm{D}_{3}$ (28), 2-(3'-hydroxypropoxy) $-1,25(\mathrm{OH})_{2} \mathrm{D}_{3}$ (29), and 2-(4'-hydroxybutoxy)-1,25$(\mathrm{OH})_{2} \mathrm{D}_{3}$ (30) (Figure 6) indicated that $\mathrm{C}-2 \beta$-substituted analogs have higher affinity for the serum vitamin $\mathrm{D}$ binding protein (DBP) and lower affinity for VDR but are superior to $\mathrm{C}-2 \alpha$ analogs in reporter gene assays. ${ }^{45}$

The analog 2-(3'-hydroxypropyl)- $1 \alpha$-methyl- $25(\mathrm{OH})_{2} \mathrm{D}_{3}$ (32) was synthesized through a Pd-catalyzed ring-closure of enyne, and coupling with vinyl bromide and showed a 2 -fold higher potency than $\mathbf{1 , 2 5}(\mathrm{OH})_{2} \mathrm{D}_{3}$ in reporter gene assays. ${ }^{46}$ Interestingly, when VDR's amino acid R274, which contacts the $1 \alpha-\mathrm{OH}$ group of the ligand (Figure 2), is mutated to a hydrophobic residue (R274L), the compound is even 7 times more potent than the natural hormone, suggesting that the $1 \alpha$ - 
<smiles>C=C1/C(=C\C)C[C@@H](O)C(CCCO)C1O</smiles><smiles>C=C1C(=CC)C[C@H](O)C(CCCCO)C1O</smiles><smiles>C=C1C(=CC)C[C@H](Cl)C(OCCO)C1O</smiles><smiles>C=C1/C(=C\C)C[C@@H](O)C(OCCCO)C1O</smiles><smiles>C=C1C(=CC)C[C@@H](O)C(OCCCO)C1O</smiles>

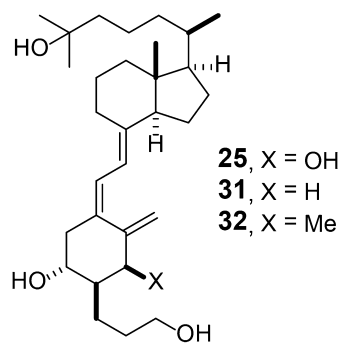

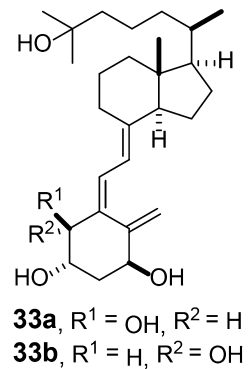

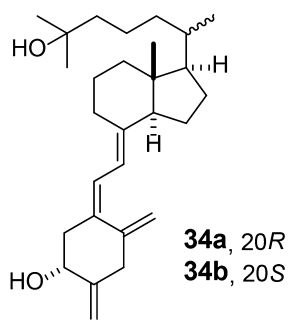<smiles>C=C1[C@@H](O)C/C(=C/C=C2\CCC[C@@]3(CCCC(C)(C)O)[C@@H](C)[C@@H]3CC[C@H]2C)C[C@H]1O</smiles>

\begin{tabular}{|c|c|c|c|c|}
\hline Family & compound & VDR aff. & $24 \mathrm{OH}$ trans & HL-60 diff \\
\hline \multirow{8}{*}{$2^{\alpha}$-subst } & 25 & ++ & - & $=$ \\
\hline & 26 & -- & -- & -- \\
\hline & 27 & $=$ & -- & $=$ \\
\hline & 28 & $=$ & - & $=$ \\
\hline & 29 & ++ & -- & $=$ \\
\hline & 30 & -- & - & -- \\
\hline & 31 & & ++ & \\
\hline & 32 & & -- & \\
\hline \multirow{2}{*}{$4-\mathrm{OH}$} & $33 a$ & - & & \\
\hline & 33b & $=$ & & \\
\hline \multirow{3}{*}{ 2-methylen } & $34 a$ & -- & -- & - \\
\hline & $34 \mathrm{~b}$ & $=$ & - & + \\
\hline & 35 & $=$ & ${ }^{++}$ & ++ \\
\hline
\end{tabular}

Figure 6. Vitamin $\mathrm{D}$ analogs with A-ring modifications. The table summarizes the biological properties of the compounds: reference, $1,25(\mathrm{OH})_{2} \mathrm{D}_{3} ;(=)$ similar value; $(+)>10 \times$ higher; $(-)>10 \times$ lower; $(--),>100 \times$ lower; VDR aff, VDR affinity; $24 \mathrm{OH}$ trans, CYP24A1 transactivation activity; HL60 diff, HL-60 cell differentiation induction.

methyl group is stabilized primarily by hydrophobic interactions.

The compounds $1 \alpha, 4 \alpha, 25(\mathrm{OH})_{3} \mathrm{D}_{3}$ (33a) and $1 \alpha, 4 \beta, 25$ $(\mathrm{OH})_{3} \mathrm{D}_{3}(33 \mathbf{b})$ were created through a Pd-catalyzed ringclosure and coupling and showed lower affinity for VDR than $\mathbf{1 , 2 5}(\mathrm{OH})_{2} \mathrm{D}_{3} \cdot{ }^{47}$ However, the $4 \beta$-analog (33b) displays higher VDR affinity and potency in reporter gene assays than the $4 \alpha$-compound (33a).

The analogs 2-methylene-25- $(\mathrm{OH}) \mathrm{D}_{3}$ (34a) and 2-methylen-20-epi-25- $(\mathrm{OH}) \mathrm{D}_{3}(34 \mathbf{b})$ belong to the family of the potent lead compound 2-methylene-1,25( $\mathrm{OH})_{2} \mathrm{D}_{3}(35,2 \mathrm{MD})$ and were synthesized through a Pd-catalyzed coupling between an enol triflate and an enyne. ${ }^{48}$ These molecules are defined by the relocation of the exocyclic methylene group from $\mathrm{C}-10$ to $\mathrm{C}-2$ and the inversion of the C-20 configuration. Compound 34a shows lower affinity for VDR than $1,25(\mathrm{OH})_{2} \mathrm{D}_{3}$, lower potency in inducing HL-60 cell differentiation and in reporter gene assays, while compound $\mathbf{3 4 b}$ displays the same affinity for VDR as $1,25(\mathrm{OH})_{2} \mathrm{D}_{3}$, higher potency in HL-60 cell differentiation induction, and lower activity in reporter gene assays.

\section{TRIENE SYSTEM MODIFICATIONS}

The compounds PRI-1731 (36), PRI-1732 (37), PRI-1733 (38), and PRI-1734 (39) represent a series of vitamin D analogs with a branched side-chain $(E)$-stereochemistry at the C-5/C-6 double bond, both configurations at C-24 and a C$22 / \mathrm{C}-23$ double bond or an $\mathrm{OH}$ group at C-22 ${ }^{49,50}$ (Figure 7). They have moderate prodifferentiating activities on HL-60 cells and their maximal inhibition of proliferation ranged from

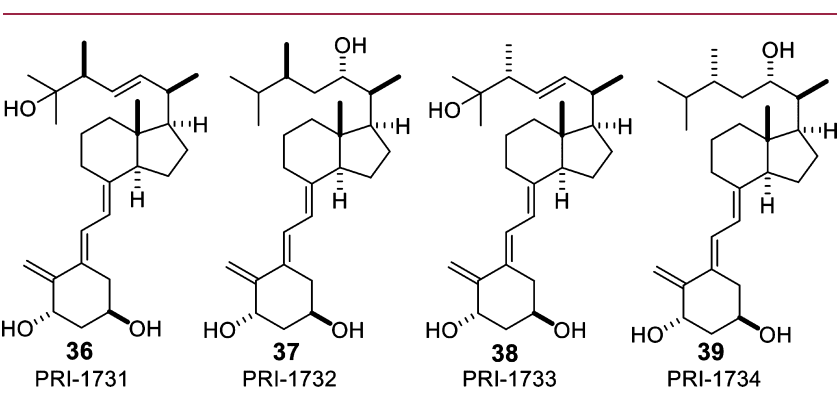

Figure 7. Triene system modified vitamin D analogs. 


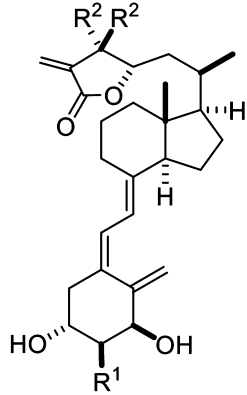

40, $R^{1}=R^{2}=H($ TEl-9647) 42, $\mathrm{R}^{1}=\mathrm{Me}, \mathrm{R}^{2}=\mathrm{H}$ 44, $\mathrm{R}^{1}=\left(\mathrm{CH}_{2}\right)_{3} \mathrm{OH}, \mathrm{R}^{2}=\mathrm{H}$ 46, $\mathrm{R}^{1}=\mathrm{O}\left(\mathrm{CH}_{2}\right)_{3} \mathrm{OH}, \mathrm{R}^{2}=\mathrm{H}$ 48, $R^{1}=H, R^{2}=M e$ 50, $\mathrm{R}^{1}=\mathrm{H}, \mathrm{R}^{2}=-\mathrm{CH}_{2} \mathrm{CH}_{2}-$ 52, $R^{1}=M e, R^{2}=M e$

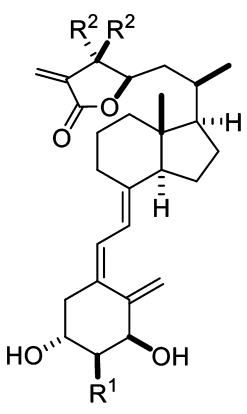

41, $R^{1}=R^{2}=H($ TEl-9648) 43, $\mathrm{R}^{1}=\mathrm{Me}, \mathrm{R}^{2}=\mathrm{H}$ 45, $\mathrm{R}^{1}=\left(\mathrm{CH}_{2}\right)_{3} \mathrm{OH}, \mathrm{R}^{2}=\mathrm{H}$ 47, $\mathrm{R}^{1}=\mathrm{O}\left(\mathrm{CH}_{2}\right)_{3} \mathrm{OH}, \mathrm{R}^{2}=\mathrm{H}$ 49, $R^{1}=H, R^{2}=M e$ 51, $\mathrm{R}^{1}=\mathrm{H}, \mathrm{R}^{2}=-\mathrm{CH}_{2} \mathrm{CH}_{2}$ 53, $\mathrm{R}^{1}=\mathrm{Me}, \mathrm{R}^{2}=\mathrm{Me}$

\begin{tabular}{|c|c|c|c|}
\hline Family & compound & VDR aff. & Antag act. \\
\hline \multirow{14}{*}{ lactone } & 40 & -- & ref \\
\hline & 42 & -- & ++ \\
\hline & 44 & -- & +++ \\
\hline & 46 & -- & ++ \\
\hline & 48 & - & + \\
\hline & 50 & ++ & ++ \\
\hline & 52 & - & ++ \\
\hline & 41 & -- & -- \\
\hline & 43 & - & - \\
\hline & 45 & - & - \\
\hline & 47 & - & - \\
\hline & 49 & - & - \\
\hline & 51 & -- & -- \\
\hline & 53 & + & + \\
\hline
\end{tabular}

Ref: $1,25(\mathrm{OH})_{2} \mathrm{D}_{3}$ :

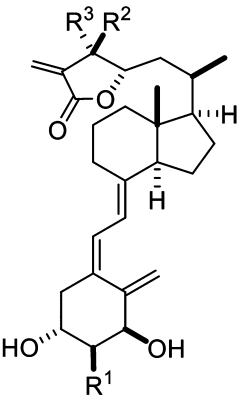

54a, $R^{1}=R^{2}=H, R^{3}=M e$ 54b, $R^{1}=M e, R^{2}=H, R^{3}=M e$

54c, $\mathrm{R}^{1}=\left(\mathrm{CH}_{2}\right)_{3} \mathrm{OH}, \mathrm{R}^{2}=\mathrm{H}, \mathrm{R}^{3}=\mathrm{Me}$

54d, $R^{1}=\mathrm{O}\left(\mathrm{CH}_{2}\right)_{3} \mathrm{OH}, \mathrm{R}^{2}=\mathrm{H}, \mathrm{R}^{3}=\mathrm{Me}$ 55a, $R^{1}=R^{2}=H, R^{3}=M e$

$55 b, R^{1}=H, R^{2}=M e, R^{3}=M e$

55c, $\mathrm{R}^{1}=\mathrm{H}, \mathrm{R}^{2}=\left(\mathrm{CH}_{2}\right)_{3} \mathrm{OH}, \mathrm{R}^{3}=\mathrm{Me}$

55d, $\mathrm{R}^{1}=\mathbf{H}, \mathrm{R}^{2}=\mathrm{O}\left(\mathrm{CH}_{2}\right)_{3} \mathrm{OH}, \mathrm{R}^{3}=\mathrm{Me}$

$56 a, R^{1}=R^{2}=H, R^{3}=E t$

$56 b, R^{1}=M e, R^{2}=H, R^{3}=E t$

56c, $\mathrm{R}^{1}=\left(\mathrm{CH}_{2}\right)_{3} \mathrm{OH}, \mathrm{R}^{2}=\mathrm{H}, \mathrm{R}^{3}=\mathrm{Et}$

56d, $\mathrm{R}^{1}=\mathrm{O}\left(\mathrm{CH}_{2}\right)_{3} \mathrm{OH}, \mathrm{R}^{2}=\mathrm{H}, \mathrm{R}^{3}=\mathrm{Et}$

57a, $R^{1}=R^{2}=H, R^{3}=E t$

57b, $R^{1}=H, R^{2}=M e, R^{3}=E t$

57c, $R^{1}=\mathrm{H}, \mathrm{R}^{2}=\left(\mathrm{CH}_{2}\right)_{3} \mathrm{OH}, \mathrm{R}^{3}=\mathrm{Et}$

57d, $R^{1}=\mathrm{H}, \mathrm{R}^{2}=\mathrm{O}\left(\mathrm{CH}_{2}\right)_{3} \mathrm{OH}, \mathrm{R}^{3}=\mathrm{Et}$

58a, $R^{1}=R^{2}=H, R^{3}=P r$

$58 b, R^{1}=M e, R^{2}=H, R^{3}=P r$

58c, $\mathrm{R}^{1}=\left(\mathrm{CH}_{2}\right)_{3} \mathrm{OH}, \mathrm{R}^{2}=\mathrm{H}, \mathrm{R}^{3}=\mathrm{Pr}$

58d, $\mathrm{R}^{1}=\mathrm{O}\left(\mathrm{CH}_{2}\right)_{3} \mathrm{OH}, \mathrm{R}^{2}=\mathrm{H}, \mathrm{R}^{3}=\mathrm{Pr}$

59a, $R^{1}=R^{2}=H, R^{3}=P r$

59b, $R^{1}=H, R^{2}=M e, R^{3}=P r$

59c, $\mathrm{R}^{1}=\mathrm{H}, \mathrm{R}^{2}=\left(\mathrm{CH}_{2}\right)_{3} \mathrm{OH}, \mathrm{R}^{3}=\mathrm{Pr}$

59d, $R^{1}=\mathrm{H}, \mathrm{R}^{2}=\mathrm{O}\left(\mathrm{CH}_{2}\right)_{3} \mathrm{OH}, \mathrm{R}^{3}=\mathrm{Pr}$

$60 a, R^{1}=R^{2}=H, R^{3}=B u$

$60 \mathrm{~b}, \mathrm{R}^{1}=\mathrm{Me}, \mathrm{R}^{2}=\mathrm{H}, \mathrm{R}^{3}=\mathrm{Bu}$

$60 \mathrm{c}, \mathrm{R}^{1}=\left(\mathrm{CH}_{2}\right)_{3} \mathrm{OH}, \mathrm{R}^{2}=\mathrm{H}, \mathrm{R}^{3}=\mathrm{Bu}$

60d, $\mathrm{R}^{1}=\mathrm{O}\left(\mathrm{CH}_{2}\right)_{3} \mathrm{OH}, \mathrm{R}^{2}=\mathrm{H}, \mathrm{R}^{3}=\mathrm{Bu}$

61a, $R^{1}=R^{2}=H, R^{3}=B u$

61b, $R^{1}=H, R^{2}=M e, R^{3}=B u$

61c, $R^{1}=\mathrm{H}, \mathrm{R}^{2}=\left(\mathrm{CH}_{2}\right)_{3} \mathrm{OH}, \mathrm{R}^{3}=\mathrm{Bu}$

61d, $\mathrm{R}^{1}=\mathrm{H}, \mathrm{R}^{2}=\mathrm{O}\left(\mathrm{CH}_{2}\right)_{3} \mathrm{OH}, \mathrm{R}^{3}=\mathrm{Bu}$

62a, $\mathrm{R}^{1}=\mathrm{R}^{2}=\mathrm{H}, \mathrm{R}^{3}=i$ - $\mathrm{Bu}$

62b, $\mathrm{R}^{1}=\mathrm{Me}, \mathrm{R}^{2}=\mathrm{H}, \mathrm{R}^{3}=i-\mathrm{Bu}$

62c, $\mathrm{R}^{1}=\left(\mathrm{CH}_{2}\right)_{3} \mathrm{OH}, \mathrm{R}^{2}=\mathrm{H}, \mathrm{R}^{3}=i-\mathrm{Bu}$

62d, $\mathrm{R}^{1}=\mathrm{O}\left(\mathrm{CH}_{2}\right)_{3} \mathrm{OH}, \mathrm{R}^{2}=\mathrm{H}, \mathrm{R}^{3}=i-\mathrm{Bu}$

63a, $\mathrm{R}^{1}=\mathrm{R}^{2}=\mathrm{H}, \mathrm{R}^{3}=i-\mathrm{Bu}$

63b, $\mathrm{R}^{1}=\mathrm{H}, \mathrm{R}^{2}=\mathrm{Me}, \mathrm{R}^{3}=i$-Bu

63c, $\mathrm{R}^{1}=\mathrm{H}, \mathrm{R}^{2}=\left(\mathrm{CH}_{2}\right)_{3} \mathrm{OH}, \mathrm{R}^{3}=i-\mathrm{Bu}$

63d, $\mathrm{R}^{1}=\mathrm{H}, \mathrm{R}^{2}=\mathrm{O}\left(\mathrm{CH}_{2}\right)_{3} \mathrm{OH}, \mathrm{R}^{3}=i-\mathrm{Bu}$

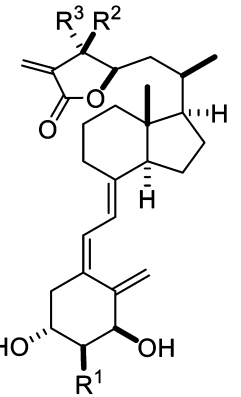

64a, $R^{1}=R^{2}=H, R^{3}=M e$

64b, $R^{1}=\mathrm{Me}, \mathrm{R}^{2}=\mathrm{H}, \mathrm{R}^{3}=\mathrm{Me}$

64c, $\mathrm{R}^{1}=\left(\mathrm{CH}_{2}\right)_{3} \mathrm{OH}, \mathrm{R}^{2}=\mathrm{H}, \mathrm{R}^{3}=\mathrm{Me}$

64d, $\mathrm{R}^{1}=\mathrm{O}\left(\mathrm{CH}_{2}\right)_{3} \mathrm{OH}, \mathrm{R}^{2}=\mathrm{H}, \mathrm{R}^{3}=\mathrm{Me}$

65a, $R^{1}=R^{2}=H, R^{3}=M e$

$65 b, R^{1}=H, R^{2}=M e, R^{3}=M e$

65c, $\mathrm{R}^{1}=\mathrm{H}, \mathrm{R}^{2}=\left(\mathrm{CH}_{2}\right)_{3} \mathrm{OH}, \mathrm{R}^{3}=\mathrm{Me}$

65d, $R^{1}=H, R^{2}=\mathrm{O}\left(\mathrm{CH}_{2}\right)_{3} \mathrm{OH}, \mathrm{R}^{3}=\mathrm{Me}$

66a, $R^{1}=R^{2}=H, R^{3}=E t$

66b, $R^{1}=M e, R^{2}=H, R^{3}=E t$

66c, $R^{1}=\left(\mathrm{CH}_{2}\right)_{3} \mathrm{OH}, \mathrm{R}^{2}=\mathrm{H}, \mathrm{R}^{3}=\mathrm{Et}$

66d, $\mathrm{R}^{1}=\mathrm{O}\left(\mathrm{CH}_{2}\right)_{3} \mathrm{OH}, \mathrm{R}^{2}=\mathrm{H}, \mathrm{R}^{3}=\mathrm{Et}$

67a, $R^{1}=R^{2}=H, R^{3}=E t$

67b, $R^{1}=H, R^{2}=M e, R^{3}=E t$

67c, $\mathrm{R}^{1}=\mathrm{H}, \mathrm{R}^{2}=\left(\mathrm{CH}_{2}\right)_{3} \mathrm{OH}, \mathrm{R}^{3}=\mathrm{Et}$

67d, $\mathrm{R}^{1}=\mathrm{H}, \mathrm{R}^{2}=\mathrm{O}\left(\mathrm{CH}_{2}\right)_{3} \mathrm{OH}, \mathrm{R}^{3}=\mathrm{Et}$

68a, $R^{1}=R^{2}=H, R^{3}=P r$

68b, $R^{1}=\mathrm{Me}, \mathrm{R}^{2}=\mathrm{H}, \mathrm{R}^{3}=\mathrm{Pr}$

$68 \mathrm{c}, \mathrm{R}^{1}=\left(\mathrm{CH}_{2}\right)_{3} \mathrm{OH}, \mathrm{R}^{2}=\mathrm{H}, \mathrm{R}^{3}=\mathrm{Pr}$

68d, $\mathrm{R}^{1}=\mathrm{O}\left(\mathrm{CH}_{2}\right)_{3} \mathrm{OH}, \mathrm{R}^{2}=\mathrm{H}, \mathrm{R}^{3}=\mathrm{Pr}$

$69 a, R^{1}=R^{2}=H, R^{3}=P r$

$69 b, R^{1}=H, R^{2}=M e, R^{3}=P r$

69c, $R^{1}=\mathrm{H}, \mathrm{R}^{2}=\left(\mathrm{CH}_{2}\right)_{3} \mathrm{OH}, \mathrm{R}^{3}=\mathrm{Pr}$

69d, $\mathrm{R}^{1}=\mathrm{H}, \mathrm{R}^{2}=\mathrm{O}\left(\mathrm{CH}_{2}\right)_{3} \mathrm{OH}, \mathrm{R}^{3}=\mathrm{Pr}$

$70 a, R^{1}=R^{2}=H, R^{3}=B u$

70b, $R^{1}=M e, R^{2}=H, R^{3}=B u$

70c, $\mathrm{R}^{1}=\left(\mathrm{CH}_{2}\right)_{3} \mathrm{OH}, \mathrm{R}^{2}=\mathrm{H}, \mathrm{R}^{3}=\mathrm{Bu}$

70d, $\mathrm{R}^{1}=\mathrm{O}\left(\mathrm{CH}_{2}\right)_{3} \mathrm{OH}, \mathrm{R}^{2}=\mathrm{H}, \mathrm{R}^{3}=\mathrm{Bu}$

71a, $R^{1}=R^{2}=H, R^{3}=B u$

71b, $R^{1}=H, R^{2}=M e, R^{3}=B u$

71c, $\mathrm{R}^{1}=\mathrm{H}, \mathrm{R}^{2}=\left(\mathrm{CH}_{2}\right)_{3} \mathrm{OH}, \mathrm{R}^{3}=\mathrm{Bu}$

71d, $\mathrm{R}^{1}=\mathrm{H}, \mathrm{R}^{2}=\mathrm{O}\left(\mathrm{CH}_{2}\right)_{3} \mathrm{OH}, \mathrm{R}^{3}=\mathrm{Bu}$

72a, $\mathrm{R}^{1}=\mathrm{R}^{2}=\mathrm{H}, \mathrm{R}^{3}=i-\mathrm{Bu}$

72b, $\mathrm{R}^{1}=\mathrm{Me}, \mathrm{R}^{2}=\mathrm{H}, \mathrm{R}^{3}=i-\mathrm{Bu}$

72c, $\mathrm{R}^{1}=\left(\mathrm{CH}_{2}\right)_{3} \mathrm{OH}, \mathrm{R}^{2}=\mathrm{H}, \mathrm{R}^{3}=i-\mathrm{Bu}$

72d, $\mathrm{R}^{1}=\mathrm{O}\left(\mathrm{CH}_{2}\right)_{3} \mathrm{OH}, \mathrm{R}^{2}=\mathrm{H}, \mathrm{R}^{3}=i-\mathrm{Bu}$

73a, $\mathrm{R}^{1}=\mathrm{R}^{2}=\mathrm{H}, \mathrm{R}^{3}=i-\mathrm{Bu}$

73b, $\mathrm{R}^{1}=\mathrm{H}, \mathrm{R}^{2}=\mathrm{Me}, \mathrm{R}^{3}=i$ - $\mathrm{Bu}$

73c, $\mathrm{R}^{1}=\mathrm{H}, \mathrm{R}^{2}=\left(\mathrm{CH}_{2}\right)_{3} \mathrm{OH}, \mathrm{R}^{3}=i-\mathrm{Bu}$

73d, $\mathrm{R}^{1}=\mathrm{H}, \mathrm{R}^{2}=\mathrm{O}\left(\mathrm{CH}_{2}\right)_{3} \mathrm{OH}, \mathrm{R}^{3}=i-\mathrm{Bu}$

Figure 8. Vitamin D analogs with side-chain and A-ring modifications (I). The table summarizes the biological properties of the compounds: reference, $1,25(\mathrm{OH})_{2} \mathrm{D}_{3} ;(=)$ similar value; $(+)>10 \times$ higher; $(++)>100 \times$ higher; $(-)>10 \times$ lower; $(--)>100 \times$ lower; VDR aff, VDR affinity; Antag act, antagonistic activity.

$10 \%$ to $15 \%$ of that for $1,25(\mathrm{OH})_{2} \mathrm{D}_{3}$ and $20-30 \%$ of that for $1,25(\mathrm{OH})_{2} \mathrm{D}_{2}$.

\section{SIDE-CHAIN AND A-RING MODIFICATIONS}

25-Dehydro- $1 \alpha$-hydroxy-vitamin $\mathrm{D}_{3}$-26,23 lactones with double modifications of C-24 and C- $2 \alpha$ were synthesized via a convergent approach by Pd-catalyzed ring closure of a enyne and subsequent coupling with a functionalized vinyl bromide. $^{51,52}$ Numerous analogs have been synthesized with variations in their $\mathrm{C}-23$ configuration, $\mathrm{C}-24$ mono- or disubstitution $[\mathrm{H}, \mathrm{Me}, \mathrm{Et}, c-\mathrm{Pr}, n-\mathrm{Pr}, n-\mathrm{Bu}, i-\mathrm{Bu}(40,41,48$,
$50,52,54,56,58,60,62,64,66,68,70,72)]$ and $\mathrm{C}-2 \alpha$ substitution $\left[\mathrm{H}, \mathrm{Me}, \mathrm{CH}_{2} \mathrm{CH}_{2} \mathrm{CH}_{2} \mathrm{OH}, \mathrm{OCH}_{2} \mathrm{CH}_{2} \mathrm{CH}_{2} \mathrm{OH}\right.$, $(42-47,49,51,53,55,57,59,61,63,65,67,69,71,73)]$ (Figure 8). The principal characteristic of these analogs is their antagonist activity, probably due to locking the VDR-LBD in a conformation where it does not effectively interact with coactivator proteins. This can be seen also from the values of the $\beta$-factors found in the crystal structure (PDB code $3 \mathrm{~A} 2 \mathrm{H}$ ) of the lactone analog TEI-9647, where the coactivator peptide shows very high values compared to $1,25(\mathrm{OH})_{2} \mathrm{D}_{3}$. In addition, helices $\mathrm{H} 9$ and 10 as well as $\mathrm{H} 11$ and to some 
<smiles>[Y]C1CC(=CC=C2CCCC3(C)CC2CCC3[2H])CC(O)CC1O</smiles>

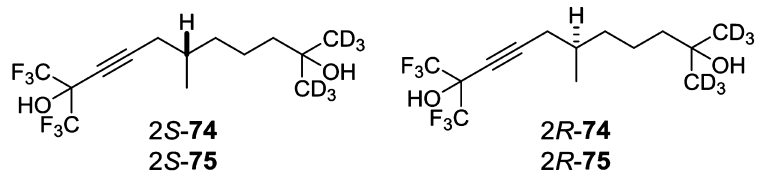<smiles>CC(CC=CC(C)(C)C)CCCC(C)(C)C</smiles><smiles>O=C(O)C=CC(C=CC(F)(F)F)CCCC(O)(O)O</smiles>

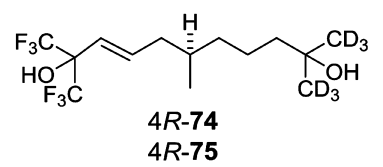<smiles>CC(C)(C)C=CCC(CCCC(C)(C)O)CCC(C)(C)C(C)(C)C</smiles>

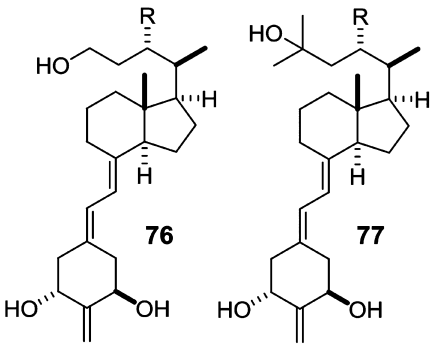

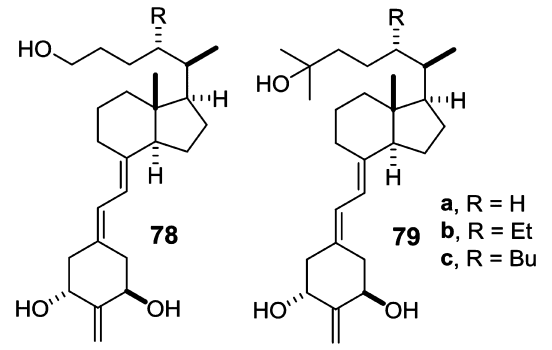

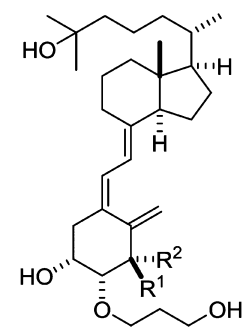

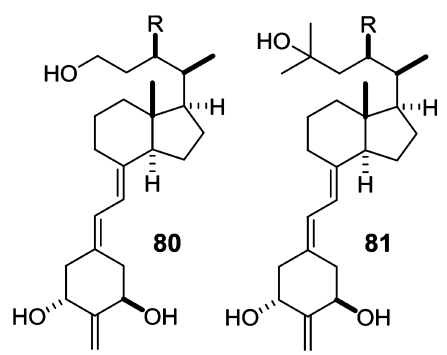<smiles>C=C1C(O)CC(=CC=C2CCC3CCC4C(C)C(CC(CC)(CC)CC)CC34C2)CC1O</smiles>

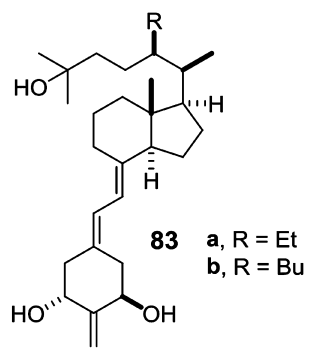

84, 20-epi-ED-71 $\mathrm{R}^{1}=\mathrm{OH}, \mathrm{R}^{2}=\mathrm{H}$

85, 1,20-epi-ED-71 $\mathrm{R}^{1}=\mathrm{H}, \mathrm{R}^{2}=\mathrm{OH}$

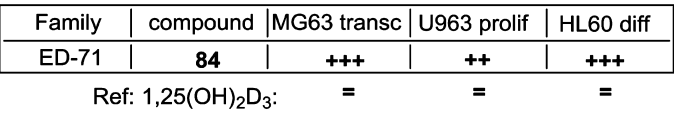

\begin{tabular}{|c|c|c|c|}
\hline Family & compound & MCF10 prol & NB4 diff \\
\hline \multirow{12}{*}{ Gemini } & $2 S-74$ & ++ & +++ \\
\hline & $2 R-74$ & ++ & +++ \\
\hline & $3 S-74$ & +++ & ++ \\
\hline & $3 R-74$ & ++ & +++ \\
\hline & $4 S-74$ & $=$ & + \\
\hline & $4 R-74$ & ++ & ++ \\
\hline & $2 S-75$ & + & ++ \\
\hline & $2 R-75$ & + & ++ \\
\hline & $3 S-75$ & ++ & +++ \\
\hline & $3 R-75$ & - & + \\
\hline & $4 S-75$ & ++ & + \\
\hline & 4R-75 & - & $=$ \\
\hline
\end{tabular}

Ref: $1,25(\mathrm{OH})_{2} \mathrm{D}_{3}$ :

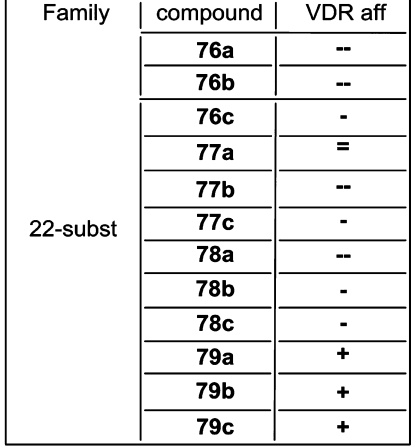

Ref: $1,25(\mathrm{OH})_{2} \mathrm{D}_{3}$ :

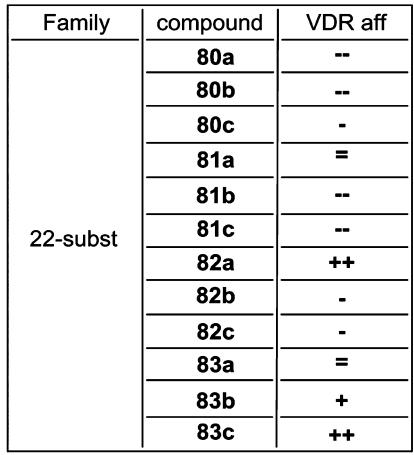

Ref: $1,25(\mathrm{OH})_{2} \mathrm{D}_{3}$ : =

Figure 9. Vitamin D analogs with side-chain and A-ring modifications (II). The table summarizes the biological properties of the compounds: reference, $1,25(\mathrm{OH})_{2} \mathbf{D}_{3} ;(=)$ similar value; $(+)>10 \times$ higher; $(++)>100 \times$ higher; $(+++)>1000 \times$ higher; $(-)>10 \times$ lower; $(-)>100 \times$ lower; VDR aff, VDR affinity; MCF10 prol, MCF10 cell proliferation inhibition; NB4 diff, NB4 cell differentiation induction; MG63 trans, MG63 cell transactivation activity; U963 prol, U963 cell proliferation inhibition; HL60 diff, HL-60 cell differentiation induction.

extent H12 show higher fluctuation leading to overall destabilization of the LBD (Figure 5B). Structure-activity relationship studies demonstrated that the exomethylene group of the lactone is indispensable, the C-23S configuration provides higher activity, and an appropriate combination of C-24 and C-2 $\alpha$ substitution obtains the highest antagonist potency.

19-nor (74) and $1 \alpha$-F (75) Gemini analogs containing triple bonds and isohexafluoro-2-propanol or isohexadeutero-2propanol side-chain end groups ${ }^{42}$ (Figure 9) were synthesized. Again $(R)$-analogs are more potent in inhibiting MCF10CA1 cell proliferation than their $(S)$-counterparts. Both isomers are active already at $100-1000$ times lower concentrations than $\mathbf{1 , 2 5}(\mathrm{OH})_{2} \mathrm{D}_{3}$. In both configurations the Gemini analogs are equally potent to $1,25(\mathrm{OH})_{2} \mathrm{D}_{3}$ in inducing $\mathrm{NB} 4$ cell differentiation but are less calcemic than the natural hormone.

A series of 2-methylene-19-nor vitamin D analogs with or without C-22S alkyl substitution, such as 2-methylene19,25,26,27-tetranor-vitamin $\mathrm{D}_{3}$ (76), 2-methylene-19,25dinor-vitamin $\mathrm{D}_{3}$ (77), 2-methylene-19,26,27-trinor-vitamin $\mathrm{D}_{3}$ (78), and 2-methylene-19-nor-vitamin $\mathrm{D}_{3}$ (79) and their C$22 \mathrm{~S}$ alkyl derivatives $(\mathrm{a}, \mathrm{R}=\mathrm{H} ; \mathbf{b}, \mathrm{R}=\mathrm{Et} ; \mathbf{c}, \mathrm{R}=\mathrm{Bu}$ ) have been prepared and biologically tested. ${ }^{53}$ The side-chain modifications in 76,77 , and 78 reduce the VDR binding affinity 10 -fold compared to $1,25(\mathrm{OH})_{2} \mathrm{D}_{3}$. Interestingly, an increasing size of the C-22 substituent in 2-methylene-19-nor-vitamin $\mathrm{D}_{3}$ (79) results in a decreased VDR binding affinity compared to $1,25(\mathrm{OH})_{2} \mathrm{D}_{3}$. Compounds with a normal side-chain (79a, 
<smiles>C=C1/C(=C\C=C2/CCCC3(CCCC(C)(C)O)C(C)CCC23)C[C@@H](O)C[C@@H]1O</smiles>

86

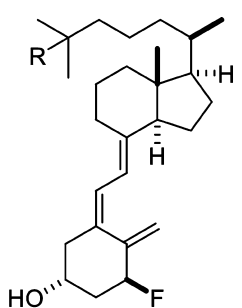

$87, \mathrm{R}=\mathrm{OH}$

88, $R=F$<smiles>C=C1/C(=C\C=C2/CCCC3(CCCC(C)(C)O)C(C)CCC23)CCCC1O</smiles>

89

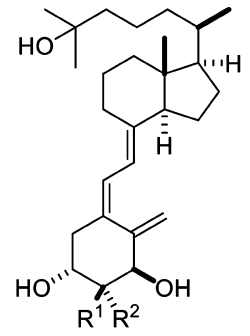

93, $R^{1}=M e, R^{2}=H$ 94, $R^{1}=H, R^{2}=M e$

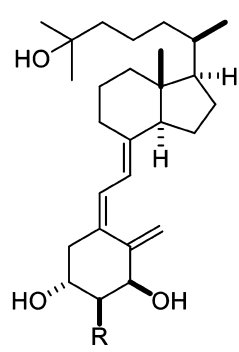

95, $\mathrm{R}=\mathrm{Et} \quad 97, \mathrm{R}^{1}=\mathrm{CH}_{2} \mathrm{CH}_{2} \mathrm{CH}_{2} \mathrm{OH}, \mathrm{R}^{2}=\mathrm{H}$ 96, $R=P r$

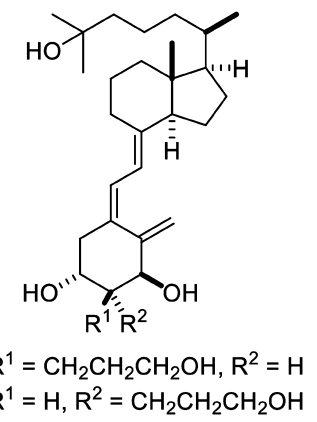

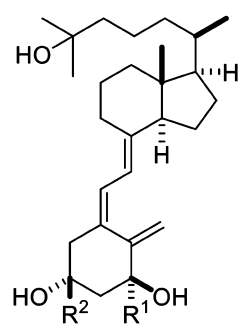

90, $\mathrm{R}^{1}=\mathrm{Me}, \mathrm{R}^{2}=\mathrm{H}$

91, $R^{1}=H, R^{2}=M e$

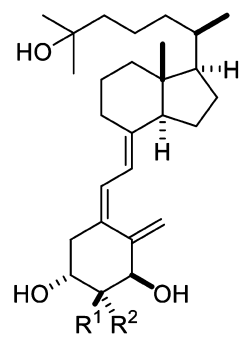

99, $\mathrm{R}^{1}=\mathrm{OCH}_{2} \mathrm{CH}_{2} \mathrm{CH}_{2} \mathrm{OH}, \mathrm{R}^{2}=\mathrm{H}$

$100, R^{1}=\mathrm{H}, \mathrm{R}^{2}=\mathrm{OCH}_{2} \mathrm{CH}_{2} \mathrm{CH}_{2} \mathrm{OH}$<smiles>C=C1/C(=C\C=C2/CCCC34C(CCC(C)(C)O)CCC(C)C3CCC24)CC(O)CC1O</smiles>

92<smiles>CC1CCCC23CCC/C(=C\C=C4C[C@@H](O)C[C@H](O)C4)C2CC[C@H]13</smiles>

101

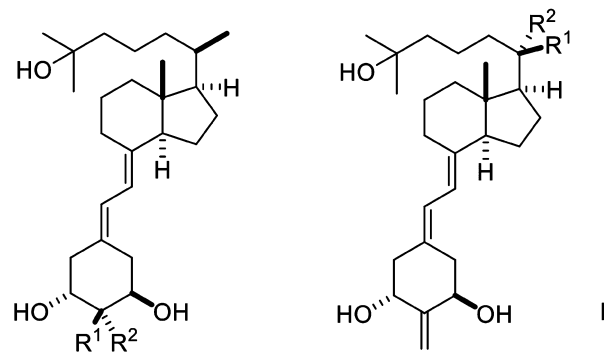<smiles>C=C1C(O)CC(=CC=C2CCCC3(C)[C@H]2CC[C@@H]3C(C)C)C[C@@H]1O</smiles><smiles>C=C1C(O)CC(=CC=C2CCCC3(C)C2CCC3C(C)CC)C[C@@H]1O</smiles>

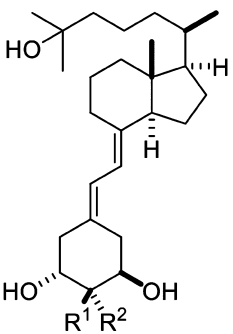

107, $\mathrm{R}^{1}=\mathrm{H}, \mathrm{R}^{2}=\mathrm{OH}$ $108, R^{1}=O H, R^{2}=H$<smiles>C/C=C1/C(O)C/C(=C\C=C2/CCC3(CCCC(C)(C)O)C2C[C@@H]2C(C)CCC23)C[C@H]1O</smiles>

109

$\begin{array}{ll}\text { 102, } R^{1}=H, R^{2}=\mathrm{OCH}_{2} \mathrm{CH}_{2} \mathrm{CH}_{2} \mathrm{OH} & 104, R^{1}=\mathrm{Me}, \mathrm{R}^{2}=\mathrm{H} \\ 103, R^{1}=\mathrm{OCH}_{2} \mathrm{CH}_{2} \mathrm{CH}_{2} \mathrm{OH}, R^{2}=\mathrm{H} & 35, R^{1}=H, R^{2}=\mathrm{Me}(2 \mathrm{MD})\end{array}$

105, 2MP

106, 2MbisP<smiles>CC1CCCC23CCC/C(=C\C=C4C[C@@H](CO)C[C@H](CO)C4)[C@H]2CC=C13</smiles>

114, QW-1624-F2-2<smiles>CCC(O)(/C=C/CC12CCC/C(=C\C=C3CC(F)C[C@H](O)C3)[C@H]1CC=C2C)CC</smiles>

115, Ro-26-9228

Figure 10. Vitamin D analogs with side-chain and A-ring modifications (III).

$79 b$, and 79c) show strong activation in reporter gene assays and compounds without C-22 substitution (76a, 77a, 78a, and 79a) even full agonist activity. In contrast, C-22S butylsubstituted molecules (76c, 76c, 78c, and 79c) present little transactivation potency, while C-22S ethyl-substituted compounds $(76 \mathrm{~b}, 77 \mathrm{~b}, 78 \mathrm{~b}$, and $79 \mathrm{~b})$ display intermediate activity. Moreover, the analogs 76a, 77a, 78a, and 79a induce the recruitment of the VDR partner receptor retinoid $\mathrm{X}$ receptor (RXR, Figure 3) and of a coactivator peptide in a concentration dependent manner, while C-22S-substituted compounds cause only moderate effects.
VDR-LBD crystal structures complexed with further 22Salkyl-2-methylene-19-nor-1,25 $(\mathrm{OH})_{2} \mathrm{D}_{3}$ derivatives $(\mathbf{8 0 - 8 3})^{54}$ confirmed that the compounds trigger the creation of an extra cavity of the LBP by rotating L305 about $27^{\circ}$ outward, in order to shelter the butyl group (Figure 5C). Ligands act as VDR antagonists when they do not interact with the C-terminal helix $\mathrm{H} 12$ of the receptor. Interestingly even though the position of helix $\mathrm{H} 12$ is maintained in the agonistic position, most likely forced by the presence of the coactivator peptide, there is a shift or rotation of multiple residues away from the 22-butyl analog. These changes weaken the interaction with H301 (loop H6/7), H393 (H11), or F418 (H12) and destabilize this 
<smiles>C=C1[C@@H](O)CC(=CC=C2CCCC3(CCCC(C)(C)O)[C@@H]2CC[C@H]3C(C)CCCC(C)(C)O)C[C@@H]1O</smiles>

104, $\mathrm{R}^{1}=\mathrm{Me}, \mathrm{R}^{2}=\mathrm{H}$ 35, $R^{1}=H, R^{2}=M e$<smiles></smiles>

119a, $R^{1}=M e, R^{2}=H$ $119 b, R^{1}=H, R^{2}=M e$

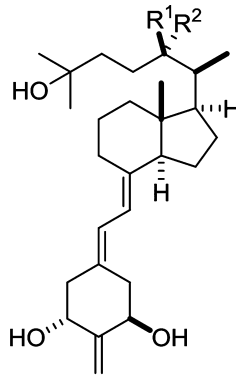<smiles>[R12]C(CCC(C)(C)O)[C@H](C)[C@H]1CC[C@H]2/C(=C/C=C3C[C@@H](O)C(=C)[C@H](O)C3)CCC[C@]21C</smiles>

116a, $R^{1}=M e, R^{2}=H \quad 117 a, R^{1}=M e, R^{2}=H$ 116b, $R^{1}=H, R^{2}=M e 117 b, R^{1}=H, R^{2}=M e$<smiles></smiles><smiles>[R1]C([R1])[C@H]1CC[C@H]2/C(=C/C=C3/C[C@@H](O)C(=C)CC3=C)CCC[C@@]2(C)[C@@H]1CCCC(C)(C)O</smiles>

$120 a, R^{1}=M e, R^{2}=H \quad 121 a, R^{1}=M e, R^{2}=H$

$120 \mathrm{~b}, R^{1}=H, R^{2}=M e 121 b, R^{1}=H, R^{2}=M e$

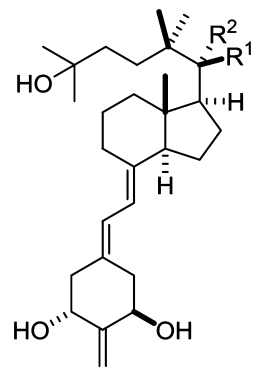

$118 a, R^{1}=M e, R^{2}=H$ $118 b, R^{1}=H, R^{2}=M e$<smiles>[R1]C1CCC2(CCCC(=CC=C3CCC(=C)C(O)C3=C)CC2)C1CCC(C)(C)O</smiles>

122a, $R^{1}=M e, R^{2}=H$ $122 b, R^{1}=H, R^{2}=M e$

\begin{tabular}{|c|c|c|c|c|}
\hline Family & compound & VDR aff & $24 \mathrm{OH}$ trans & HL60 diff \\
\hline \multirow{13}{*}{ 2-methylen } & $116 a$ & - & -- & - \\
\hline & $116 \mathrm{~b}$ & $=$ & ++ & $=$ \\
\hline & $117 a$ & ++ & +++ & +++ \\
\hline & $117 b$ & -- & - & -- \\
\hline & $118 a$ & ++ & ++ & - \\
\hline & $119 a$ & -- & -- & -- \\
\hline & $119 b$ & -- & - & - \\
\hline & $120 a$ & -- & $=$ & + \\
\hline & $120 b$ & - & + & + \\
\hline & $121 a$ & -- & -- & -- \\
\hline & $121 b$ & - & - & -- \\
\hline & $122 a$ & -- & $=$ & + \\
\hline & $122 b$ & - & $=$ & + \\
\hline
\end{tabular}

Figure 11. Vitamin D analogs with side-chain and A-ring modifications (IV). The table summarizes the biological properties of the compounds: reference, $1,25(\mathrm{OH})_{2} \mathbf{D}_{3} ;(=)$ similar value; $(+)>10 \times$ higher; $(++)>100 \times$ higher; $(+++)>1000 \times$ higher; $(-)>10 \times$ lower; $(--)>100 \times$ lower; VDR aff, VDR affinity; 24OH trans, CYP24A1 transactivation activity; HL60 diff, HL-60 cell differentiation induction.

region of the LBD (Figure 5C). Interestingly, in the presence of a coactivator peptide some of the antagonistic 22-butyl analogs take the agonistic conformation. Whether this is a technical artifact of the crystallization or has a physiological meaning, such as sensing of cofactor balance in the cellular context, needs to be clarified.

20-epi-Eldecalcitol (84), a 20-epi derivative of the antiosteoporotic drug eldecalcitol (12) (Table 1 and Figure 1 ), was synthesized through a convergent approach by Pdcatalyzed ring-closure of an enyne and coupling with vinyl bromide. $^{55}$ Since 20 -epi-1,25 $(\mathrm{OH})_{2} \mathrm{D}_{3}$, a diasteromer of $1,25(\mathrm{OH})_{2} \mathrm{D}_{3}$ possessing an inverted C-21 methyl-substituent at $\mathrm{C}-20$, shows enhanced biological activities compared to $1,25(\mathrm{OH})_{2} \mathrm{D}_{3}$, compound 84 displays a 50-fold increased inhibition of U937 human leukemia cell proliferation. ${ }^{56}$
Since the presence of a $1 \alpha-\mathrm{OH}$ group in $1,25(\mathrm{OH})_{2} \mathrm{D}_{3}$ is crucial for VDR binding, its replacement with a $1 \beta$-OH group $\left[1 \beta, 25(\mathrm{OH})_{2} \mathrm{D}_{3}, 86\right]$ causes loss of physiological activity ${ }^{57}$ (Figure 10). Replacing the $1 \alpha-\mathrm{OH}$ group with one fluor atom $\left[1 \alpha-\mathrm{F}, 25(\mathrm{OH})_{2} \mathrm{D}_{3}, 87\right]$ also markedly diminishes biological activity, ${ }^{58}$ while a compound with each a fluorine atom at C-1 and C-25 $\left[1,25(\mathrm{~F})_{2}(\mathrm{OH})_{2} \mathrm{D}_{3}, \mathbf{8 8}\right]$ is devoid of all activity. ${ }^{59}$ Interestingly, the $3-\mathrm{OH}$ group is not necessary if the $1 \alpha-\mathrm{OH}$ group is already in position, but the lack of the $3-\mathrm{OH}$ group $\left[1 \alpha, 25(\mathrm{OH})_{2}-3\right.$-deoxy- $\left.\mathrm{D}_{3}, 89\right]$ reduces the biological activity. ${ }^{6,61}$ Additional methyl groups at $\mathrm{C}-1 \beta[1 \beta$-methyl-1,25$\left.(\mathrm{OH})_{2} \mathrm{D}_{3}, 90\right]$ and $\mathrm{C}-3 \alpha\left[3 \alpha\right.$-methyl-1,25 $\left.(\mathrm{OH})_{2} \mathrm{D}_{3}, 91\right]$ significantly reduce VDR binding affinity. Switching the 3$\mathrm{OH}$ group from $\beta$ to $\alpha$ position [3-epi-1,25( $\mathrm{OH})_{2} \mathrm{D}_{3}, 92$ ] causes a drastic reduction of physiological activity. ${ }^{62}$ In fact, 


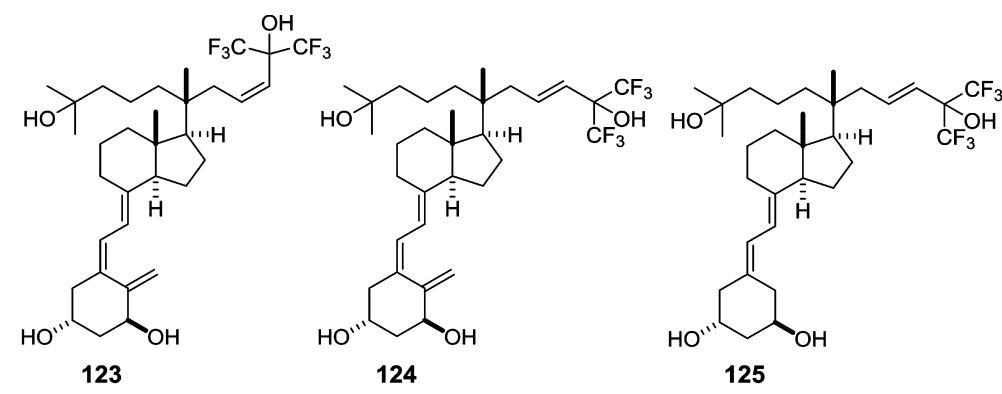

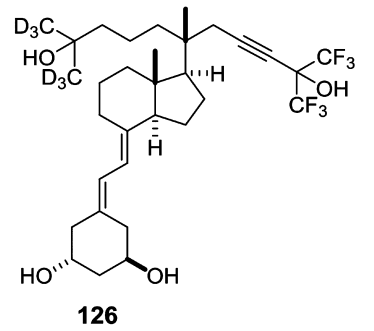

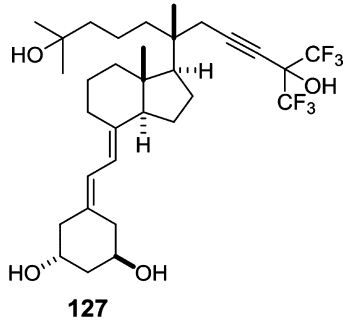<smiles>[R]C1[C@H](O)C=C(C#CC2=CCCC34CCC[C@H](C)[C@@H]3CC[C@@H]24)C[C@H]1O</smiles>

128, $\mathrm{R}=\mathrm{H}, \mathrm{H}$ 129, $\mathrm{R}=\mathrm{CH}_{2}$

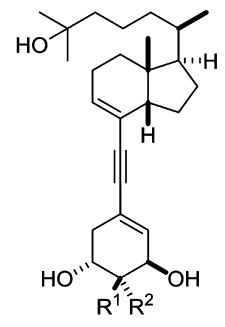

130, $\mathrm{R}^{1}=\alpha-\mathrm{CH}_{3}, \mathrm{R}^{2}=\mathrm{H}$ $131, R^{1}=H, R^{2}=\beta-\mathrm{CH}_{3}$
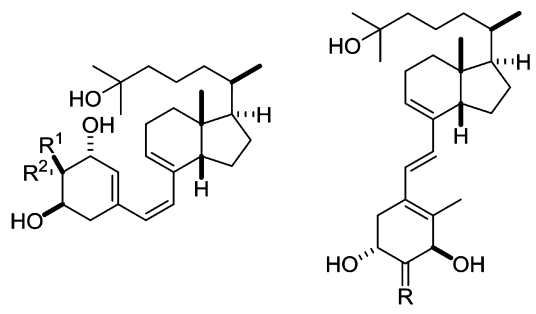

132. $\mathrm{R}^{1}=\mathrm{H} \mathrm{R}^{2}=\mathrm{H}$ 133, $R^{1}=\alpha-\mathrm{CH}_{3}, \mathrm{R}^{2}=\mathrm{H}$ 134, $R^{1}=H, R^{2}=\beta-C_{3}$
135, $\mathrm{R}=\mathrm{H} \mathrm{H}$ 136, $\mathrm{R}=\alpha-\mathrm{CH}_{3}, \mathrm{H}$ $137, \mathrm{R}=\mathrm{H}, \mathrm{\beta}-\mathrm{CH}_{3}$

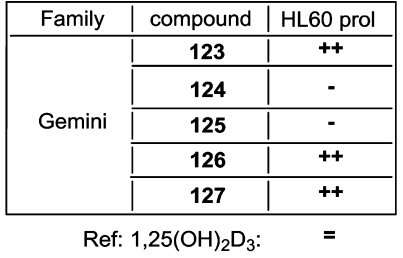

\begin{tabular}{|c|c|c|}
\hline Family & compound & VDR aff \\
\hline \multirow{7}{*}{ 14-epi } & 129 & - \\
\cline { 2 - 3 } & 130 & - \\
\cline { 2 - 3 } & 131 & -- \\
\cline { 2 - 3 } & 132 & -- \\
\cline { 2 - 3 } & 133 & -- \\
\cline { 2 - 3 } & 134 & -- \\
\hline & 135 & -- \\
\hline & 136 & $=$ \\
\hline 137 & - \\
\hline
\end{tabular}

Ref: $1,25(\mathrm{OH})_{2} \mathrm{D}_{3}: \quad=$

Figure 12. Vitamin $\mathrm{D}$ analogs with side-chain and A-ring modifications $(\mathrm{V})$. The table summarizes the biological properties of the compounds: reference, $1,25(\mathrm{OH})_{2} \mathbf{D}_{3} ;(=)$ similar value; $(+)>10 \times$ higher; $(++)>100 \times$ higher; $(-)>10 \times$ lower; $(--)>100 \times$ lower; VDR aff, VDR affinity; HL60 diff, HL-60 cell differentiation induction.

compound 92 is an intermediate of $\mathbf{1 , 2 5}(\mathrm{OH})_{2} \mathrm{D}_{3}$ degradation displaying lower VDR binding affinity and lower calcemic effects compared to $\mathbf{1 , 2 5}(\mathbf{O H})_{2} \mathrm{D}_{3}$. Despite its decreased potency, the in vivo action of compound $\mathbf{9 2}$ is tissue-specific.

Taken together, most modifications of the A-ring result in decreased biological activity except for those modified at C-2. Therefore, a large number of C-2-substituted vitamin D analogs have been synthesized and were studied intensively for their biological activity. $2 \alpha$-Substitutions [ $2 \alpha$-methyl-1,25$\left.(\mathrm{OH})_{2} \mathrm{D}_{3}(93)\right]$ are more potent than $2 \beta$-substitutions $[2 \beta$ methyl-1,25(OH) $\left.{ }_{2} \mathrm{D}_{3}(\mathbf{9 2})\right]^{63}$ Elongation of the C-2-alkyl group $[2 \alpha \text {-ethyl-1,25(OH })_{2} \mathrm{D}_{3}$ (94) and $2 \alpha$-propyl-1,25$\left.(\mathrm{OH})_{2} \mathrm{D}_{3}(95)\right]$ reduces VDR binding affinity and biological potency, but $\omega$-hydroxylation restores the activity. ${ }^{64} 2 \alpha$ Methyl-1,25(OH $)_{2} \mathrm{D}_{3}(\mathbf{9 3})$ is twice as calcemic as 1,25$(\mathrm{OH})_{2} \mathbf{D}_{3}$. In combination with 20 -epimerization [ $2 \alpha$-methyl20-epi-1,25( $\left.\mathrm{OH})_{2} \mathrm{D}_{3}\right]$ VDR binding affinity increases even 12 fold. ${ }^{63} 2 \alpha$-(3'-Hydroxypropyl)-1,25(OH $)_{2} \mathrm{D}_{3}$ (97) has a 3 -fold increased VDR binding affinity than $1,25(\mathrm{OH})_{2} \mathrm{D}_{3}$, while $2 \beta$ (3'-hydroxypropyl)-1,25(OH) ${ }_{2} \mathrm{D}_{3}$ (98) is 1.4 times more potent. Terminal hydroxylation of $2 \alpha$ - and $2 \beta$-propoxy groups at C-2, $2 \alpha$-hydroxypropoxy- $1,25(\mathrm{OH})_{2} \mathrm{D}_{3}(99)$ and $2 \beta$ hydroxypropoxy-1,25( $\mathrm{OH})_{2} \mathrm{D}_{3}(\mathbf{1 0 0})$ also increases the VDR binding potential.

19-nor-Vitamin D analogs are known to be devoid of hypercalcemic and hyperphosphatemic effects. ${ }^{65,66}$ For example, 19-nor-1,25(OH) ${ }_{2} \mathrm{D}_{3}$ (101) has a 5 times reduced the VDR binding affinity compared to $1,25(\mathrm{OH})_{2} \mathrm{D}_{3}$ paired with low or no bone calcification activity, while 19-nor-1,25$(\mathrm{OH})_{2} \mathrm{D}_{2}$ (paricalcitol, 9, Figure 1) has similar affinity as the natural hormone. Selected modifications at the C-2 position of 19-nor-vitamin $\mathrm{D}$ analogs are more potent inducers of gene activity. $2 \beta$-(3'-Hydroxypropoxy)-19-nor- $1,25(\mathrm{OH})_{2} \mathrm{D}_{3}$ (102) and $2 \alpha$-( $3^{\prime}$-hydroxypropoxy)-19-nor-1,25 $(\mathrm{OH})_{2} \mathrm{D}_{3}(103)$ have reduced potency in bone and intestine. ${ }^{67} 2$-Methylene-19-nor20-epi-1,25( $\mathrm{OH})_{2} \mathrm{D}_{3}(\mathbf{2 M D}, 35)$ shows VDR binding affinity comparable to $1,25(\mathrm{OH})_{2} \mathrm{D}_{3}$ but a 100 times enhanced ability to mobilize calcium from bone. ${ }^{68}$ Moreover, $2 \mathrm{MD}$ is 10 times more potent than $\mathbf{1 , 2 5}(\mathrm{OH})_{2} \mathbf{D}_{3}$ in activating CYP24A1 gene activity and inducing HL-60 cell differentiation. Analogs of 2MD with a shortened side-chain, such as $2 \mathrm{MP}$ (105) and 2MbisP (106) are able to reduce parathyroid hormone (PTH) production. $^{69}$

The compound 19-nor- $1 \alpha, 2 \beta, 25(\mathrm{OH})_{3} \mathrm{D}_{3}(107)$ is as potent as $1,25(\mathrm{OH})_{2} \mathrm{D}_{3}$ in intestinal calcium transport, while its epimer 19-nor- $1 \alpha, 2 \alpha, 25(\mathrm{OH})_{3} \mathrm{D}_{3}$ (108) possesses less activity. $^{70}$ Importantly, both compounds are not calcemic. Compound 107 has higher VDR binding affinity than 108 and is more potent in inhibiting MCF-7 cell proliferation. When the 2-ethylidene group is in E-configuration (109), VDR binding affinity is 2.4-fold increased. Moreover, a $3^{\prime}$ hydroxypropylidene group at C-2 in E-configuration resulted in the potent compounds AGR (110) and 1AGS (111), which seem to be intestine-selective. ${ }^{60}$

$2 \alpha$-(3'-Hydroxypropyl)-19-nor-1,25( $\mathrm{OH})_{2} \mathrm{D}_{3}$ (112), which is modified at both $\mathrm{C}-2$ and $\mathrm{C}-10$, shows a similar VDR binding affinity as $\mathbf{1 , 2 5}(\mathrm{OH})_{2} \mathrm{D}_{3}$, displays a 36 -fold higher potential in inducing HL-60 cell differentiation, and has a 500fold higher antiproliferative potency in PZ-HPV-7 prostate cells. Its epimer $2 \beta$-( $3^{\prime}$-hydroxypropyl)-19-nor-1,25( $\left.\mathrm{OH}\right)_{2} \mathrm{D}_{3}$ 

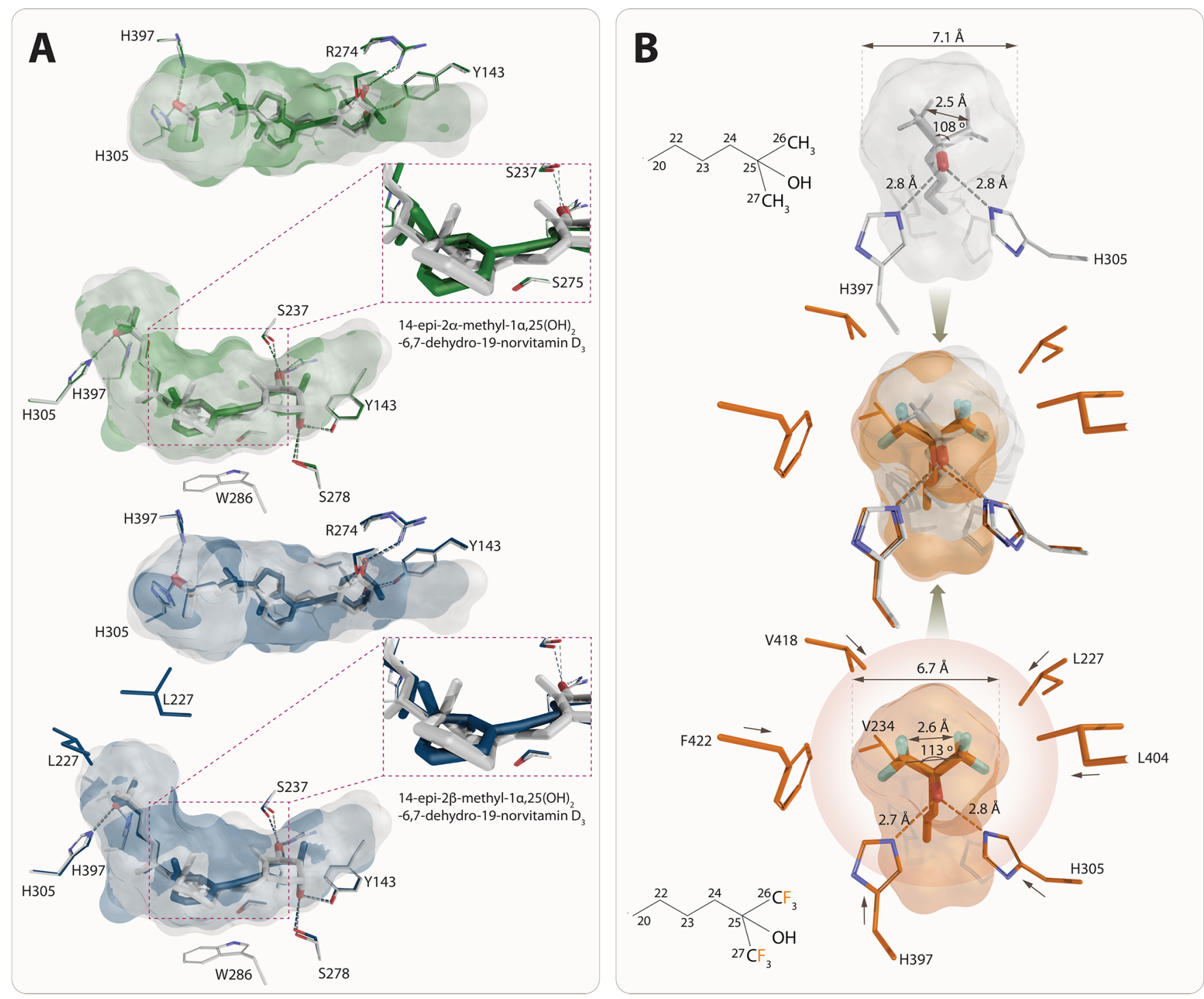

Figure 13. Structure-function relationship of various VDR ligands (II). The binding of 14-epi-2 $\alpha$-methyl- and 14-epi-2 $\beta$-methyl-1,25(OH) 2 -6,7dehydro-19-norvitamin $\mathrm{D}_{3}$. The overall spatial conservation of the $\mathrm{OH}$ groups is maintained, but the CD-ring structure for the epimers shows higher flexibility by changing the more planar conformation in $\mathbf{1 , 2 5}(\mathrm{OH})_{2} \mathrm{D}_{3}$ to reverse V-letter shape. This is largely due to the bending of the Cring downward. The other notable changes involve the proximal location of L227 in $2 \beta$-methyl epimer and W286 for $1,25(\mathrm{OH})_{2} \mathrm{D}_{3}$ under $3.5 \AA$ cutoff. Structural elements from 14-epi-2 $\alpha$-methyl- (PDB code 3AUQ), 14-epi-2 $\beta$-methyl-1,25(OH) ${ }_{2}$-6,7-dehydro-19-norvitamin $\mathrm{D}_{3}$ (PDB code 3AUR), and 1,25( $\mathrm{OH})_{2} \mathrm{D}_{3}$ (PDB code 1DB1) complexes are highlighted in green, blue, and white color, respectively (A). Possible mechanism governing the potency of fluorinated analogs. The effect of a fluorinated functional group is illustrated on $\mathrm{CF}_{3}$ group in comparison with $\mathrm{CH}_{3}$ group located at the terminal carbons C-26 and C-27 of the ligand's aliphatic chain. The high electronegativity of the fluor atom has a pulling effect for the hydrophobic residues located in the proximity of the functional group (bottom), which cannot be seen for the $1,25(\mathrm{OH})_{2} \mathrm{D}_{3}$ under the same $3.5 \AA$ cutoff (top). However, the hydrogen bonds between the conserved histidines and the $25-\mathrm{OH}$ group are maintained in both scenarios. The positions of the $\mathrm{CF}_{3}$ groups show a moderate opening about $5^{\circ}$ with maintained distance between carbon $\mathrm{C}-26$ and $\mathrm{C}-27$. These changes have effect on the LBP, which is a bit smaller (middle panel) in the presence of $\mathrm{CF}_{3}$ functional groups (B). Structural elements for the $\mathrm{CF}_{3}$ and $\mathrm{CH}_{3}$ groups are highlighted in orange and white color, respectively.

(113) has lower VDR binding and prodifferentiation activity, but it is as potent as $\mathbf{1 1 0}$ in inhibiting prostate cell proliferation. $^{70,71}$

Promising analogs with three different alterations in the vitamin $\mathrm{D}$ skeleton (the A-ring, the side-chain, and the $\mathrm{CD}$ ring) are $1 \beta$-hydroxymethyl-16-ene-24,24- $\mathrm{F}_{2}-26,27$-bishomo$25(\mathrm{OH})_{2} \mathrm{D}_{3}(\mathrm{QW}-1624-\mathrm{F} 2-2,114)^{72}$ and $1 \alpha$-fluor-16-ene-20epi-23-ene-26,27-bishomo-25(OH $)_{2} \mathrm{D}_{3}$ (Ro-26.9228, 115). ${ }^{73}$ In a skin cancer model compound 114 inhibits progression and molecule 115 restores bone loss, while both are not hypercalcemic.
Six new derivatives of compound 104 have been prepared by a convergent synthesis using the Wittig-Horner approach ${ }^{74}$ in order to evaluate the influence of methyl groups at C-22 on biological activity (Figure 11). Single methylation of the (20R)-25-hydroxylated side-chain (117) did not change the VDR binding affinity in comparison to the parent compound 104. However, the addition of a 22-methyl group to the (20S)25-hydroxylated side-chain (118) caused a much stronger effect. The $22 R$-compound 118 a has a 2.5 times higher VDR binding affinity than 104 and is 250 -fold more potent than its 22-epimer $118 \mathbf{b}$. The prodifferentiation potential of an analog 


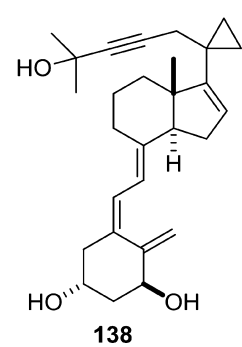

138

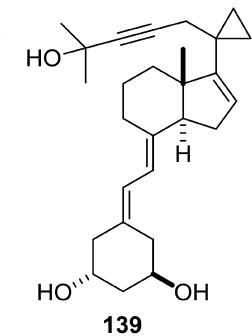

139

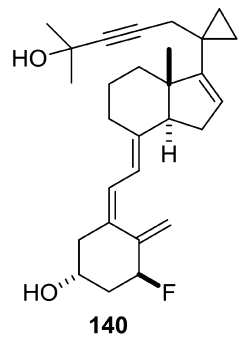

140<smiles>C=C1/C(=C\C=C2/CCCC3(C)C(C#CC(C)(C)O)CCC23)CCCC1O</smiles>

141

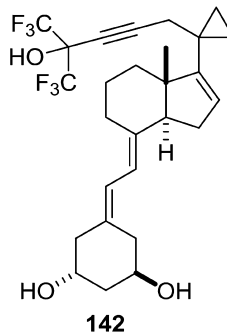

142

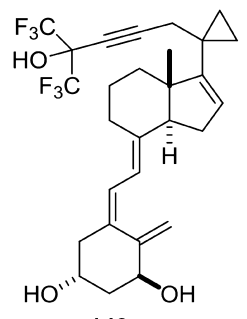

143

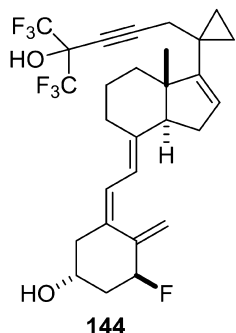

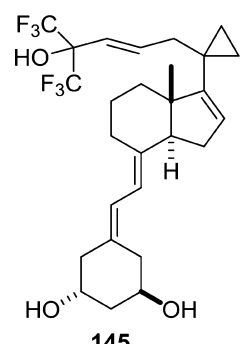<smiles></smiles><smiles>C=C1C(=CC=C2CCCC3(C)C2C=CC3C2(CC=CC(C)(O)C(F)(F)F)CC2)C[C@@H](O)C[C@H]1F</smiles><smiles></smiles><smiles>C=C1/C(=C\C=C2/CCCC3(C)C(C4(CCC(C)(C)O)CC4)=CCC23)C[C@@H](O)C[C@@H]1O</smiles><smiles>C=C1C(=CC=C2CCCC3(C)C(C4(CCC(=O)C(C)(C)O)CC4)=CCC23)C[C@@H](O)CC1O</smiles>

\begin{tabular}{|c|c|c|c|}
\hline Family & compound & MLR IFN- $\gamma$ & LPS TNF- $\alpha$ \\
\hline \multirow{12}{*}{ Gemini } & 138 & $=$ & +++ \\
\hline & 139 & $=$ & +++ \\
\hline & 140 & + & -- \\
\hline & 141 & -- & \\
\hline & 142 & + & -- \\
\hline & 143 & $=$ & + \\
\hline & 144 & -- & \\
\hline & 145 & + & -- \\
\hline & 146 & $=$ & -- \\
\hline & 147 & - & - \\
\hline & 148 & $=$ & $=$ \\
\hline & 149 & +++ & +++ \\
\hline
\end{tabular}

Figure 14. A-ring, D-ring, and side-chain modified vitamin $D$ analogs. The table summarizes the biological properties of the compounds: reference, $1,25(\mathrm{OH})_{2} \mathrm{D}_{3} ;(=)$ similar value; $(+)>10 \times$ higher; $(+++)>1000 \times$ higher; $(-)>10 \times$ lower; $(--)>100 \times$ lower; MLR IFN $-\gamma$, INFG inhibition; LPS TNF $\alpha$, TNF inhibition.

with a $22 S$-methyl group in the "natural" side-chain (20R) (116a) is 10-fold higher than that of its 22-epimer (116b), whereas in the case of "unnatural" $20 S$-compound the $22 R$ epimer $(\mathbf{1 1 7 a})$ is 1000 times more potent than its 22-epimer $(\mathbf{1 1 7 b})$ and 4 -fold more potent than the parent compound 104. When two methyl groups were introduced at C-22, such as in the 20R-compound (118a) and the 20S-compound (118b), VDR binding affinity is increased compared to their parent molecules.

C-20-isomers of 25( $\mathrm{OH})$-2-methylene-vitamin $\mathrm{D}_{3}$ and 3desoxy-1 $\alpha, 25(\mathrm{OH})_{2}$-2-methylene-vitamin $\mathrm{D}_{3}(117-120)$ were synthesized through a convergent approach using a Sonorogashira coupling ${ }^{75}$ (Figure 11). The biological activities of compounds 119-122 are clearly lower than those of the parent compound 104. With the exception of the $1 \alpha$ hydroxylated compounds they were also less active than $1,25(\mathrm{OH})_{2} \mathrm{D}_{3}$. Analogs without a $1 \alpha-\mathrm{OH}$ group show lower VDR binding affinity, HL-60 cell prodifferentiation activity, and CYP24A1 activation than those hydroxylated at C-1. The addition of the 10-exo-methylene group improved the in vitro activity of the (20S)-1-desoxy compounds. In contrast, in the (20S)-series only VDR binding affinity augmented. The presence of the 2-exomethylene group resulted in enhanced intestinal calcium transport compared to $1,25(\mathrm{OH})_{2} \mathrm{D}_{3}$, but bone calcium mobilization was 10 -fold decreased in the (20R)series.

A large structure-function analysis of 39 Gemini derivatives ${ }^{43}$ showed five compounds $(123-127)$ with enhanced antiproliferative activity (Figure 12). Compound 127 was stronger than $1,25(\mathrm{OH})_{2} \mathrm{D}_{3}$ in inhibiting cancer cell growth, while both were equipotent in their calcemic effect.

The Sonogashira approach was used to synthesize novel 14epi derivatives of 19 -nor- $1 \alpha, 25(\mathrm{OH})_{2}$-previtamin $\mathrm{D}_{3}$ (132) and 19 -nor- $1 \alpha, 25(\mathrm{OH})_{2}$-tachysterol $\mathrm{D}_{3} \quad(135){ }^{76}$ Dienynic compounds (129-131) showed moderate VDR binding affinity, where the 2-methylene compound (129) has higher activity than the 2-methyl-substituted diastereomers 130 and 131. Previtamin $D_{3}$ compounds (132-134) showed low VDR binding affinity. 14-epi-19-nor-tachysterol) $\mathrm{D}_{3}$ compounds displayed higher VDR binding affinity, of which 2-methylene14-epi-19-nor-tachysterol $\mathrm{D}_{3}$ (137) is most potent. Crystal structure analysis indicated unique binding conformations. The binding of both epimers, 14-epi-2 $\alpha$-methyl- (PDB code $3 A U Q)$ and 14-epi-2 $\beta$-methyl-1,25( $\mathrm{OH})_{2}$-6,7-dehydro-19-nor- 
<smiles>[R]c1ccc(CCCCC(CC)(CC)CC)cc1</smiles>

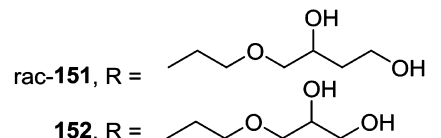

$153, R=$<smiles></smiles><smiles>[R]/C=C(/C=C1C[C@@H](O)C[C@H](O)C1=C)c1cccc(CCCCCC(C)(C)O)c1</smiles>

166a, $R=E t$

166b, $R=n-P r$

166c, $R=n-B u$

166d, $R=n-H e x$

166e, $R=n-H e p$

\begin{tabular}{|c|c|c|c|c|c|}
\hline & comp & VDR aff & $24 \mathrm{OH}$ tr & MCF7 pr & $\mathrm{Ca}$ \\
\hline \multirow{5}{*}{$\begin{array}{l}\text { triene } \\
\text { hybrids }\end{array}$} & $166 a$ & - & - & $=$ & -- \\
\hline & $166 \mathrm{~b}$ & -- & -- & $=$ & - \\
\hline & $166 \mathrm{c}$ & - & -- & + & - \\
\hline & $166 \mathrm{~d}$ & - & -- & + & - \\
\hline & $166 \mathrm{e}$ & - & - & + & - \\
\hline
\end{tabular}

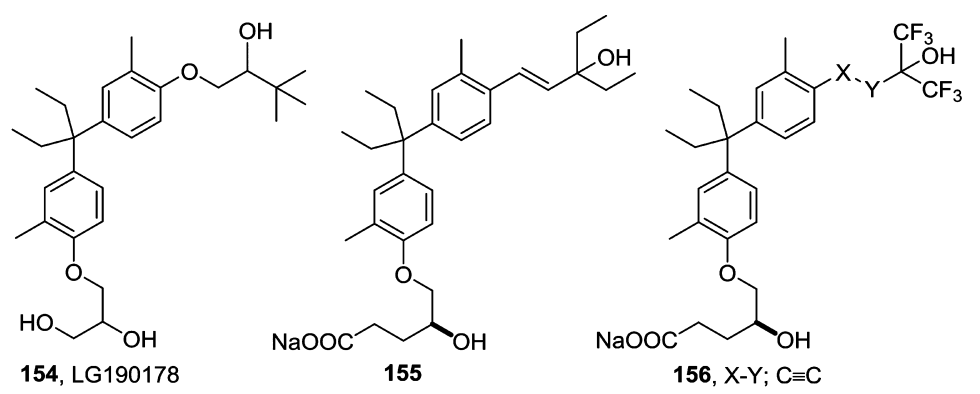

$157, \mathrm{X}-\mathrm{Y} ;(E)-\mathrm{CH}=\mathrm{CH}$

158, X-Y; $\mathrm{CH}_{2} \mathrm{CH}_{2}$

\begin{tabular}{|c|c|c|c|}
\hline Family & compound & VDR aff & HL60 diff \\
\hline \multirow{3}{*}{ carboranyl } & rac-151 & & -- \\
\cline { 2 - 4 } & 152 & -- & +++ \\
\hline \multirow{4}{*}{$\begin{array}{c}\text { diphenyl } \\
\text { hybrids }\end{array}$} & 153 & -- & - \\
\cline { 2 - 4 } & 154 & + & + \\
\cline { 2 - 4 } & 155 & $=$ & $=$ \\
\cline { 2 - 4 } & 156 & - & ++ \\
\cline { 2 - 4 } & 158 & ++ & ++ \\
\hline \multicolumn{2}{|c|}{ Ref: $1,25(\mathrm{OH})_{2} \mathrm{D}_{3}:$} & $=$ & $=$ \\
\hline
\end{tabular}

160a, $\mathrm{X}=\mathrm{N}, \mathrm{R}^{1}=\mathrm{H}, \mathrm{R}^{2}=\left(\mathrm{CH}_{2}\right)_{3} \mathrm{~N}\left(\mathrm{CH}_{3}\right)_{2}$ 160b, $\mathrm{X}=\mathrm{N}, \mathrm{R}^{1}=\mathrm{H}, \mathrm{R}^{2}=\left(\mathrm{CH}_{2}\right)_{3} \mathrm{~N}\left(\mathrm{CH}_{2} \mathrm{CH}_{3}\right)_{2}$ 160c, $X=\mathrm{N}, \mathrm{R}^{1}=\mathrm{H}, \mathrm{R}^{2}=\left(\mathrm{CH}_{2}\right)_{2} \mathrm{~N}\left(\mathrm{CH}_{3}\right)_{2}$

$\begin{array}{ll}160 c, X=N, R^{1}=H, R^{2}=\left(C_{2}\right)_{2} N_{(}\left(C_{3}\right)_{2} & 162 c, X=N, R^{1}=H, R^{2}=P h-m-O_{3} F_{3} \\ \left.160 d, X=N, R^{1}=H, R^{2}=\left(C_{2}\right)_{2} N_{(} C_{2} C_{3}\right)_{2} & 162 d, X=N, R^{1}=H, R^{2}=P h-p-O_{2} C_{2} C_{3}\end{array}$ 160e, $X=\mathrm{N}, \mathrm{R}^{1}=\mathrm{H}, \mathrm{R}^{2}=\mathrm{CH}_{2} \mathrm{CN}$ 160f, $X=N, R^{1}=H, R^{2}=\left(\mathrm{CH}_{2}\right)_{2} \mathrm{NH}_{2}$ 160g, $X=\mathrm{N}, \mathrm{R}^{1}=\mathrm{H}, \mathrm{R}^{2}=\left(\mathrm{CH}_{2}\right)_{3} \mathrm{~N}$ 160h, $X=\mathrm{N}, \mathrm{R}^{1}=\left(\mathrm{CH}_{2}\right)_{2} \mathrm{CN}, \mathrm{R}^{2}=\left(\mathrm{C}_{2}\right)_{2} \mathrm{CN}$ 160i, $X=\mathrm{N}, \mathrm{R}^{1}=\mathrm{CH}_{3}, \mathrm{R}^{2}=\mathrm{CH}_{2} \mathrm{CH}=\mathrm{CH}_{2}$ 161a, $X=O, R^{1}=\mathrm{CH}\left(\mathrm{CH}_{3}\right) \mathrm{C}=\mathrm{CH}$ 161b, $\mathrm{X}=\mathrm{O}, \mathrm{R}^{1}=\left(\mathrm{CH}_{2}\right)_{2} \mathrm{C} \equiv \mathrm{CH}$ 162a, $\mathrm{X}=\mathrm{N}, \mathrm{R}^{1}=\mathrm{Ph}-p-\mathrm{CH}_{2} \mathrm{CO}_{2} \mathrm{CH}_{3}$

162b $X=N, R^{1}=H, R^{2}=P h-p-O F^{2}$

163a, $X=\mathrm{N}, \mathrm{R}^{1}=\mathrm{H}, \mathrm{R}^{2}=\mathrm{CH}\left[\mathrm{CH}\left(\mathrm{CH}_{3}\right) \mathrm{C}_{2} \mathrm{H}_{5}\right] \mathrm{CO}_{2} \mathrm{CH}_{3}$

163b, $X=\mathrm{N}, \mathrm{R}^{1}=\mathrm{CH}_{3}, \mathrm{R}^{2}=\mathrm{CH}_{2} \mathrm{CO}_{2} \mathrm{CH}_{2} \mathrm{CH}_{3}$

163c, $X=\mathrm{N}, \mathrm{R}^{1}=\mathrm{H}, \mathrm{R}^{2}=\mathrm{CH}\left(\mathrm{CO}_{2} \mathrm{CH}_{3}\right) \mathrm{CH}_{2} \mathrm{CO}_{2} \mathrm{CH}_{3}$

164a, $X=N, R^{1}=\mathrm{H}, \mathrm{R}^{2}=\mathrm{CH}\left[\mathrm{CH}\left(\mathrm{CH}_{3}\right) \mathrm{C}_{2} \mathrm{H}_{5}\right] \mathrm{CO}_{2} \mathrm{H}$

164b, $X=\mathrm{N}, \mathrm{R}^{1}=\mathrm{CH}_{3}, \mathrm{R}^{2}=\mathrm{CH}_{2} \mathrm{CO}_{2} \mathrm{H}$

164c, $X=\mathrm{N}, \mathrm{R}^{1}=\mathrm{H}, \mathrm{R}^{2}=\mathrm{CH}\left(\mathrm{CO}_{2} \mathrm{CH}_{3}\right) \mathrm{CO}_{2} \mathrm{H}$

164d, $X=\mathrm{N}, \mathrm{R}^{1}=\mathrm{H}, \mathrm{R}^{2}=\mathrm{CH}\left(\mathrm{CH}_{3}\right) \mathrm{CO}_{2} \mathrm{H}$

165a, $X=\mathrm{N}, \mathrm{R}^{1}=\mathrm{H}, \mathrm{R}^{2}=\mathrm{CH}\left[\mathrm{CH}\left(\mathrm{CH}_{3}\right) \mathrm{C}_{2} \mathrm{H}_{5}\right] \mathrm{CH}_{2} \mathrm{OH}$

165b, $X=\mathrm{N}, \mathrm{R}^{1}=\mathrm{CH}_{3}, \mathrm{R}^{2}=\mathrm{CH}_{2} \mathrm{CH}_{2} \mathrm{OH}$

165c, $X=\mathrm{N}, \mathrm{R}^{1}=\mathrm{H}, \mathrm{R}^{2}=\mathrm{CH}\left(\mathrm{CH}_{2} \mathrm{OH}\right) \mathrm{CH}_{2} \mathrm{CH}_{2} \mathrm{OH}$

165d, $X=\mathrm{N}, \mathrm{R}^{1}=\mathrm{H}, \mathrm{R}^{2}=\mathrm{CH}\left(\mathrm{CH}_{3}\right) \mathrm{CH}_{2} \mathrm{OH}$

\begin{tabular}{|c|c|c|c|c|c|c|c|}
\hline Family & compound & HL60 diff & MCF7 prol & Family & compound & HL60 diff & MCF7 prol \\
\hline \multirow{14}{*}{$\begin{array}{l}\text { pyrrolyl } \\
\text { phenyl } \\
\text { hybrids }\end{array}$} & $160 a$ & + & +++ & \multirow{14}{*}{$\begin{array}{l}\text { pyrrolyl } \\
\text { phenyl } \\
\text { hybrids }\end{array}$} & 162d & -- & -- \\
\hline & $160 b$ & $=$ & ++ & & $163 a$ & -- & $=$ \\
\hline & $160 c$ & -- & ++ & & $163 b$ & -- & + \\
\hline & $160 d$ & +++ & -- & & $163 c$ & - & +++ \\
\hline & $160 \mathrm{e}$ & -- & ++ & & $164 a$ & -- & - \\
\hline & $160 f$ & - & +++ & & $164 b$ & - & +++ \\
\hline & $160 \mathrm{~g}$ & -- & -- & & $164 c$ & - & - \\
\hline & $160 \mathrm{~h}$ & -- & -- & & $164 d$ & - & - \\
\hline & $160 \mathrm{i}$ & -- & -- & & $165 a$ & -- & - \\
\hline & $161 a$ & --- & -- & & $165 b$ & - & ++ \\
\hline & $161 b$ & -- & -- & & $165 c$ & -- & - \\
\hline & $162 a$ & -- & -- & & $165 d$ & - & - \\
\hline & $162 b$ & -- & -- & & & & \\
\hline & $162 c$ & -- & - & & & & \\
\hline
\end{tabular}

Ref: $1,25(\mathrm{OH})_{2} \mathrm{D}_{3}$ :

Figure 15. Nonsteroidal VDR ligands. The table summarizes the biological properties of the compounds: reference, $1,25(\mathbf{O H}){ }_{2} \mathrm{D}_{3} ;(=)$ similar value; $(+)>10 \times$ higher; $(++)>100 \times$ higher; $(+++)>1000 \times$ higher; $(-)>10 \times$ lower; $(--)$, >100 $\times$ lower; $(---)$, >1000× lower; VDR aff, VDR affinity; calcemia, $\left[\mathrm{Ca}^{2+}\right]$ level changes in serum; $24 \mathrm{OH}$ trans, CYP24A1 transactivation activity; HL60 diff, HL-60 cell differentiation induction; MCF7 pr, MCF7 proliferation inhibition.

vitamin $\mathrm{D}_{3}$ (PDB code 3AUR) is very similar in maintaining the position of the anchoring $\mathrm{OH}$ groups seen from the $\mathbf{1 , 2 5}(\mathrm{OH})_{2} \mathrm{D}_{3}$ complex (PDB code $\left.1 \mathrm{DB} 1\right)$. However, the modification between $\mathrm{C}-6$ and $\mathrm{C}-7$ provides rigidity for this 
region introducing an unforeseen compensation in flexibility for the $\mathrm{CD}$ rings. Compared to their more planar conformation in $\mathbf{1 , 2 5}(\mathrm{OH})_{2} \mathrm{D}_{3}$, here they take a reverse V-letter conformation such as by the bending of the $\mathrm{C}$ ring about $9^{\circ}$ downward. For the $2 \beta$-methyl isomer the residue L227 is closer to the ligand under $3.5 \AA$ cutoff but under similar cutoff W286 seems to be closer to $1,25(\mathbf{O H})_{2} D_{3}$, which is due to more planar conformation of the CD-rings (Figure 13A).

\section{SIDE-CHAIN, D-RING, AND A-RING MODIFICATIONS}

The synthesis of 12 analogs $(138-149)$ of $1 \alpha, 25(\mathrm{OH})_{2}-16-$ ene-20-cyclopropylvitamin $\mathrm{D}_{3}$ relied on Wittig-Horner coupling $^{77}$ (Figure 14). These compounds have an unsaturated D-ring between $\mathrm{C}-16$ and $\mathrm{C}-17$ and a cyclopropyl group located at C-20. The structural diversity on the side-chain covered triple CC bonds (138-144), double CC bonds (145-147) and single $C C$ bonds (148 and 149) together with the A-ring covered $1 \alpha, 3 \beta$-dihydroxy $(138,143,146$, and 149), 19 -nor (139, 142, 145, and 148), 3-deoxy-1 $\alpha$-hydroxy (141), and $1 \alpha$-fluor-3 $\beta$-hydroxy (140 and 144). The anti-inflammatory properties of these compounds were studied via analyzing the inhibition of the secretion of the cytokines interferon- $\gamma$ (IFNG) and tumor necrosis factor (TNF). Most of 16-ene-20cyclopropyl analogs inhibited IFNG with similar potency to $1,25(\mathrm{OH})_{2} \mathrm{D}_{3}$, but compound 149 was more potent. The inhibition of TNF showed wide differences, some analogs (138, 140, 143-145) failed to induce TNF inhibition, whereas analog 149 inhibited TNF more efficiently than $1,25(\mathrm{OH})_{2} \mathrm{D}_{3}$. The metabolism of 149 was studied and the stable 24-oxo metabolite 150 accumulated during metabolism. Compound 150 mediates similar induction of primary vitamin $\mathrm{D}$ target genes as analog 149 but has a lower calcemic activity.

\section{NONSTEROIDAL VDR LIGANDS}

The synthesis of nonsteroidal VDR agonists containing a hydrophobic 1,12-dicarba-closo-dodecaborane ( $p$-carborane) unit was achieved through bimolecular nucleophilic substitution $^{78}$ (Figure 15). The carborane cage replaced the CD-rings of the natural hormone exploiting the hydrophobicity of $p$ carborane. Despite their simple and flexible structure, the carborane-based VDR ligands show moderate binding affinity for VDR compared to $1,25(\mathrm{OH})_{2} \mathrm{D}_{3}$. The analogs are flexible acyclic triols; i.e., they lack an A-ring and conjugated triene structures. Their structures shared a branched side-chain on a carborane carbon, and in the other carbon three different chains are bound either to 3-oxaheptan-5,7-diol (151), 3oxahexan-5,6-diol (152) or 4-oxaheptan-6,7-diol (153). The flexibility of the diol is favorable for VDR binding affinity, which, however, is more then 100 times lower than for $\mathbf{1 , 2 5}(\mathrm{OH})_{2} \mathrm{D}_{3}$. Nevertheless, these mimics are rather active in inducing HL-60 cell differentiation [rac-151, 5\%; $(S)-151,8 \%$; (R)-151, 2\%; 152, 0.05\%; 153, 0.001\%)]. The $S$-isomers showed, compared to the $R$-enantiomer, higher prodifferentiation activity and VDR binding affinity.

LG190178 (154) is the first published nonsteroidal vitamin $\mathrm{D}$ analog. ${ }^{79}$ In general, VDR ligands based on bisphenyl core compounds with $\gamma$-hydroxycarboxylic acid moiety (155) show agonist activity. From compounds with a fluorine-containing bisphenyl core ${ }^{80}$ the hexafluoro analog (157) is 5 times more potent in reporter gene assays than the parent compound (155), shows 2 times higher prodifferentiation activity, and is 7 times more effective in inducing bone $\gamma$-carboxyglutamate protein $(B G L A P)$ expression. Like in secosteroidal vitamin D analogs, fluorination is an effective modification as shown by crystal structure analysis of the VDR-LBD complexed with 155. Also in this case helix $\mathrm{H} 12$ is stabilized in the agonistic position allowing interaction with coactivator proteins. From physicochemical point of view the fluorine atom's ionic radius is $100 \%$ larger than that of the hydrogen, the van der Waals radius is only $27 \%$ larger. However, the high electronegativity of fluor has a possible "pulling" effect for residues residing in the nearest proximity of a fluorinated functional group. This can be illustrated on the comparison of the aliphatic chain of the ligands that have $\mathrm{CH}_{3}$ or $\mathrm{CF}_{3}$ functional groups at carbon $\mathrm{C}-26$ and $\mathrm{C}-27$. The latter shows additional five hydrophobic residues that cannot be seen for $\mathrm{CH}_{3}$ at the cutoff $3.5 \AA$ (Figure 13B). The only maintained interactions are with conserved histidine residues. In addition, the possible reaction to the strong van der Waals forces from hydrophobic residues is a moderate opening of the functional groups by $5^{\circ}$ with maintained distance between carbon C-26 and C-27 due to additional twist in the absolute position of the $\mathrm{C}-25$ carbon. These net effects show also small variation the LBP size, which is slightly confined in the presence of $\mathrm{CF}_{3}$ functional groups resulting in a tighter packing of the cavity (Figure 13B).

Nonsteroidal vitamin $\mathrm{D}$ mimics with phenylpyrrolyl pentane skeletons have been designed (159-165) ${ }^{81,82}$ Among them, 159 shows clear antiproliferative effects on MCF-7 cells. In order to improve the biological activity of compound 159, derivatives were designed comprising side-chains terminated in a diethylcarbinol, hydrophilic groups or hydrophobic groups (160-165). The antiproliferative activities of the compounds were tested in MCF-7 cells, PC3 human prostate cancer cells, Caco2 human colon cancer cells, and HepG2 human liver cancer cells. Compound $160 \mathrm{~b}$ exhibits the best antiproliferative activity, being more potent than the prototype compound 159 and $1,25(\mathrm{OH})_{2} \mathrm{D}_{3}$. Also the compounds 160a, 160c, 160d, $160 \mathrm{f}, 160 \mathrm{~g}, 164 \mathrm{~b}$, and $165 \mathrm{~b}$ show in all four model systems better antiproliferative activities than 159 and $1,25(\mathrm{OH})_{2} \mathrm{D}_{3}$. The $\mathrm{R}^{2}$ substitutions at the pyrrole-ring side-chains are crucial for the antiproliferative activity of the compounds. Molecules with hydrophilic groups at the end of the pyrrole-ring sidechain (160a, 160b, 160d, 160g, and 164b) are more potent than those bearing hydrophobic groups (161a, 161b, 162a-d, 163a, and 164b). Moreover, compounds 160a, 160c, 160d, $160 \mathrm{~g}$, and 164b were less cytotoxic than 159 and 1,25$(\mathrm{OH})_{2} \mathrm{D}_{3}$. Compounds $160 \mathrm{a}-\mathrm{d}, 164 \mathrm{~b}$, and $165 \mathrm{~b}$ also display prodifferentiating activity. In reporter gene assays $164 \mathrm{~b}$ is the most potent compound, whereas the transactivation potential of $160 \mathrm{~b}$ and $160 \mathrm{~g}$ is comparable to that of $1,25(\mathrm{OH})_{2} \mathrm{D}_{3}$.

A novel class of analogs, ${ }^{83}$ where the C-ring and D-ring were replaced by an aromatic $m$-phenylene $\mathrm{D}$-ring and an alkyl chain, were synthesized based on the formation of the triene system through a Pd-catalyzed ring-closure of an enol trifate and a subsequent Suzuki-Miyaura reaction with appropriate boronate in aqueous medium. ${ }^{84}$ Compounds 166a-e efficiently induce the differentiation of human keratinocytes and show antiproliferative activity in MCF-7, PC-3, SKOV-3 (human ovary cancer), and $\mathrm{HaCaT}$ cells comparable to $1,25(\mathrm{OH})_{2} \mathrm{D}_{3}$. Compound $166 \mathrm{a}$ with the shortest chain at $\mathrm{C}-8$ is most active not only in antiproliferative tests but also in reporter gene assays. Importantly, none of compounds 166a-e induce hypercalcemia. In a SCID mice xenograph model of aggressive MDA-MB-231 human breast cancer cells compound 
166a shows high efficacy for tumor growth inhibition and overall survival.

\section{CONCLUSIONS}

This review demonstrated that clever and relevant chemistry significantly increased the number and variety of synthetic vitamin $\mathrm{D}$ analogs. Analog design had advanced and led to functional molecules, such as the $o$-carborane compounds, that are devoid of a 25-OH group. Moreover, there are now molecules that completely lack A- and/or CD-rings, such as $p$ carborane compounds, but still interact with VDR. Some of these nonsteroidal vitamin $\mathrm{D}$ analogs display high activity in vitro in combination with low calcemic effects in vivo. Thus, the area of nonsteroidal analogs and mimics is expected to further rise in future.

The assessment of the biological profile of VDR ligands is still primarily reduced to in vitro assays, such as VDR binding affinity, reporter gene assays, and antiproliferative and prodifferentiation measurements in different cancer cell lines. The variety in the assays makes a direct comparison of the different types of vitamin D analogs difficult. Moreover, a reliable extrapolation of the in vivo potential of the compounds is impossible without changing to a different set of assays, such as gene expression profiles in freshly isolated human peripheral blood mononuclear cells. ${ }^{85}$

Nowadays research on vitamin $\mathrm{D}$ analogs is nearly exclusively performed in academia and many interesting approaches for optimizing the profile of VDR ligands have not been explored to their limits. Accordingly, a complete picture is still missing and there is potential for improvements. The number of nearly 150 solved crystal structures of the VDR-LBD complexed with synthetic ligands is impressive and demonstrates the active interest of academia in understanding the molecular actions of VDR agonists and antagonists.

Unfortunately, failures of clinical trails focused on cancer have majorly dampened the interest of pharma industry in further developing vitamin $\mathrm{D}$ compounds. Since the natural hormone $1,25(\mathrm{OH})_{2} \mathrm{D}_{3}$ primarily prevents bone- and immunesystem-related diseases, the molecule and it synthetic derivatives may not be perfect drugs for the therapy of cancer. Nevertheless, calcipotriol-activated VDR in stroma of human pancreatic tumors had been shown to markedly reduce markers of inflammation and fibrosis in pancreatitis and human tumor stroma. ${ }^{86}$ This suggests that vitamin D compounds rather affect immune cells of the microenvironment of tumors than directly inhibiting the proliferation of the cancer cells. In fact, to date, most genome-wide data on the action of VDR and its ligands are available from cells of the hematopoietic system. ${ }^{87}$ This further emphasizes the impact of vitamin $\mathrm{D}$ and VDR for innate and adaptive immunity and suggests that these areas should be further explored for a commercial application.

\section{ASSOCIATED CONTENT}

\section{S Supporting Information}

The Supporting Information is available free of charge on the ACS Publications website at DOI: 10.1021/acs.jmedchem.9b00208.

Table S1 describing all 143 publically available VDR ligand crystal structures with individual hyperlinks to the PDB and PubMed databases and citations of refs 90-144 (PDF)

\section{AUTHOR INFORMATION}

\section{Corresponding Author}

*Phone: +358-40-355-3062. E-mail: carsten.carlberg@uef.fi.

ORCID $\odot$

\section{Carsten Carlberg: 0000-0003-2633-0684}

\section{Notes}

The authors declare no competing financial interest.

\section{Biographies}

Miguel A. Maestro received his Ph.D. in Chemistry from the University of Santiago de Compostela (Spain) in 1989. He did his postdoctoral stay in synthetic organic chemistry at the ETH-Zentrum (Zürich, Switzerland). In 1991 he joined the Faculty of Sciences at the University of A Coruña (Spain) studying synthetic methodologies towards vitamin D metabolites and analogs. Since 2018 he holds a Professor position at the Department of Chemistry. Prof. Maestro's interests are the synthesis of new vitamin D analogs with isotopic labeling and the atomic disposition of molecular structures through $\mathrm{X}$ ray crystallography.

Ferdinand Molnár received his Ph.D. in Biochemistry from the University of Kuopio (Kuopio, Finland) in 2006. He did his postdoctoral training in Structural Biology at the IGBMC (Illkirch, France). In 2008 he joined the School of Pharmacy at the University of Eastern Finland (Kuopio, Finland) studying nuclear receptorligand, -protein, and -DNA interactions. In 2018 he moved to the Nazarbayev University (Astana, Kazakhstan) where he holds an Associate Professor position at the Department of Biology. Prof. Molnár's interests are integrative structural biology and bioinformatics, eukaryotic transcriptional regulation in health and disease, and recombinant protein production.

Carsten Carlberg graduated in 1989 with a Ph.D. in Biochemistry at the Free University Berlin (Germany). After positions as postdoc at Roche (Basel, Switzerland), group leader at the University of Geneva (Switzerland), and docent at the University of Düsseldorf (Germany) he is since 2000 Full Professor of Biochemistry at the University of Eastern Finland in Kuopio (Finland). His work focuses on mechanisms of gene regulation by nuclear hormones, in particular on vitamin D. At present Prof. Carlberg has projects on epigenomewide effects of vitamin $\mathrm{D}$ on the human immune system.

\section{ACKNOWLEDGMENTS}

M.A.M. thanks Xunta de Galicia (Grant ED431B-2018/ GI2105) for financial support. C.C. thanks the Academy of Finland for support.

\section{ABBREVIATIONS USED}

$\mathbf{1 , 2 5}(\mathrm{OH})_{2} \mathrm{D}_{3}, \quad 1 \alpha, 25$-dihydroxyvitamin $\mathrm{D}_{3} ; \mathbf{1 , 2 5}(\mathrm{OH})_{2} \mathrm{D}_{2}$, $1 \alpha$,25-dihydroxyvitamin $\mathrm{D}_{2} ; 25(\mathrm{OH}) \mathrm{D}_{3}$, 25-hydroxyvitamin $\mathrm{D}_{3}$; BGLAP, bone $\gamma$-carboxyglutamate protein (previously called osteocalcin); CAMP, cathelicidin antimicrobial peptide; CYP24A1, cytochrome P450, family 24, subfamily A, polypeptide 1; DBP, vitamin D binding protein; IFNG, interferon- $\gamma$; LBD, ligand-binding domain; LBP, ligand-binding pocket; PDB, Protein Data Base; PTH, parathyroid hormone; $\mathrm{RXR}$, retinoid $\mathrm{X}$ receptor; TNF, tumor necrosis factor; TSS, transcription start site; VDR, vitamin $\mathrm{D}$ receptor

\section{REFERENCES}

(1) Tremezaygues, L.; Sticherling, M.; Pfohler, C.; Friedrich, M.; Meineke, V.; Seifert, M.; Tilgen, W.; Reichrath, J. Cutaneous photosynthesis of vitamin $\mathrm{D}$ : an evolutionary highly-conserved endocrine system that protects against environmental hazards 
including UV-radiation and microbial infections. Anticancer Res. 2006, 26 (4A), 2743-2748.

(2) Jasinghe, V. J.; Perera, C. O.; Barlow, P. J. Bioavailability of vitamin $\mathrm{D}_{2}$ from irradiated mushrooms: an in vivo study. Br. J. Nutr. 2005, 93 (6), 951-955.

(3) Norman, A. W. From vitamin D to hormone D: fundamentals of the vitamin $\mathrm{D}$ endocrine system essential for good health. Am. J. Clin. Nutr. 2008, 88 (2), 491S-499S.

(4) Hollis, B. W. Circulating 25-hydroxyvitamin D levels indicative of vitamin D sufficiency: implications for establishing a new effective dietary intake recommendation for vitamin D. J. Nutr. 2005, 135 (2), 317-322.

(5) Carlberg, C.; Polly, P. Gene regulation by vitamin $\mathrm{D}_{3}$. Crit. Rev. Eukaryotic Gene Expression 1998, 8 (1), 19-42.

(6) Bouillon, R.; Suda, T. Vitamin D: calcium and bone homeostasis during evolution. BoneKEy Rep. 2014, 3, 480.

(7) Hewison, M. An update on vitamin D and human immunity. Clin. Endocrinol. (Oxford, U. K.) 2012, 76 (3), 315-325.

(8) Rook, G. A. The role of vitamin D in tuberculosis. Am. Rev. Respir. Dis. 1988, 138 (4), 768-770.

(9) Ramagopalan, S. V.; Maugeri, N. J.; Handunnetthi, L.; Lincoln, M. R.; Orton, S. M.; Dyment, D. A.; Deluca, G. C.; Herrera, B. M.; Chao, M. J.; Sadovnick, A. D.; Ebers, G. C.; Knight, J. C. Expression of the multiple sclerosis-associated MHC class II Allele HLADRB1*1501 is regulated by vitamin D. PLoS Genet. 2009, 5 (2), e1000369.

(10) Holick, M. F.; Binkley, N. C.; Bischoff-Ferrari, H. A.; Gordon, C. M.; Hanley, D. A.; Heaney, R. P.; Murad, M. H.; Weaver, C. M. Evaluation, treatment, and prevention of vitamin D deficiency: an Endocrine Society clinical practice guideline. J. Clin. Endocrinol. Metab. 2011, 96 (7), 1911-1930.

(11) Carlberg, C. The physiology of vitamin D-far more than calcium and bone. Front. Physiol. 2014, 5, 335.

(12) Holick, M. F. Vitamin D deficiency. N. Engl. J. Med. 2007, 357, 266-281.

(13) Kupferschmidt, K. Uncertain verdict as vitamin D goes on trial. Science 2012, 337 (6101), 1476-1478.

(14) Institute of Medicine. Dietary Reference Intakes for Calcium and Vitamin D; Ross, A. C., Taylor, C. L., Yaktine, A. L., Del Valle, H. B., Eds.; National Academies Press: Washington, DC, 2011.

(15) Cheskis, B. J.; Freedman, L. P.; Nagpal, S. Vitamin D receptor ligands for osteoporosis. Curr. Opin Invest. Drugs 2006, 7 (10), 906911.

(16) Fogh, K.; Kragballe, K. New vitamin D analogs in psoriasis. Curr. Drug Targets: Inflammation Allergy 2004, 3 (2), 199-204.

(17) Bouillon, R.; Okamura, W. H.; Norman, A. W. Structurefunction relationships in the vitamin D endocrine system. Endocr. Rev. 1995, 16, 200-257.

(18) Carlberg, C.; Mouriño, A. New vitamin D receptor ligands. Expert Opin. Ther. Pat. 2003, 13, 761-772.

(19) Carlberg, C.; Molnár, F.; Mouriño, A. Vitamin D receptor ligands: the impact of crystal structures. Expert Opin. Ther. Pat. 2012, 22 (4), 417-435.

(20) Evans, T. R.; Colston, K. W.; Lofts, F. J.; Cunningham, D.; Anthoney, D. A.; Gogas, H.; de Bono, J. S.; Hamberg, K. J.; Skov, T.; Mansi, J. L. A phase II trial of the vitamin D analogue Seocalcitol (EB1089) in patients with inoperable pancreatic cancer. Br. J. Cancer 2002, 86 (5), 680-685.

(21) Heikkinen, S.; Väisänen, S.; Pehkonen, P.; Seuter, S.; Benes, V.; Carlberg, C. Nuclear hormone $1 \alpha, 25$-dihydroxyvitamin $\mathrm{D}_{3}$ elicits a genome-wide shift in the locations of VDR chromatin occupancy. Nucleic Acids Res. 2011, 39 (21), 9181-9193.

(22) Gombart, A. F.; Borregaard, N.; Koeffler, H. P. Human cathelicidin antimicrobial peptide (CAMP) gene is a direct target of the vitamin $\mathrm{D}$ receptor and is strongly up-regulated in myeloid cells by 1,25-dihydroxyvitamin $D_{3}$. FASEB J. 2005, 19 (9), 1067-1077.

(23) Haussler, M. R.; Haussler, C. A.; Jurutka, P. W.; Thompson, P. D.; Hsieh, J. C.; Remus, L. S.; Selznick, S. H.; Whitfield, G. K. The vitamin D hormone and its nuclear receptor: molecular actions and disease states. J. Endocrinol. 1997, 154 (Suppl.), S57-S73.

(24) Carlberg, C. Vitamin D genomics: from in vitro to in vivo. Front. Endocrinol. 2018, 9, 250.

(25) Verstuyf, A.; Carmeliet, G.; Bouillon, R.; Mathieu, C. Vitamin D: a pleiotropic hormone. Kidney Int. 2010, 78 (2), 140-145.

(26) Wang, Y.; Zhu, J.; DeLuca, H. F. Where is the vitamin D receptor? Arch. Biochem. Biophys. 2012, 523 (1), 123-133.

(27) Carlberg, C.; Molnár, F. Vitamin D receptor signaling and its therapeutic implications: genome-wide and structural view. Can. J. Physiol. Pharmacol. 2015, 93, 311-318.

(28) Rochel, N.; Wurtz, J. M.; Mitschler, A.; Klaholz, B.; Moras, D. Crystal structure of the nuclear receptor for vitamin D bound to its natural ligand. Mol. Cell 2000, 5, 173-179.

(29) Molnár, F. Structural considerations of vitamin D signaling. Front. Physiol. 2014, 5, 191.

(30) Molnár, F.; Peräkylä, M.; Carlberg, C. Vitamin D receptor agonists specifically modulate the volume of the ligand-binding pocket. J. Biol. Chem. 2006, 281 (15), 10516-10526.

(31) Carlberg, C. Molecular basis of the selective activity of vitamin D analogues. J. Cell. Biochem. 2003, 88 (2), 274-281.

(32) Carlberg, C. Molecular endocrinology of vitamin D on the epigenome level. Mol. Cell. Endocrinol. 2017, 453, 14-21.

(33) Wei, Z.; Yoshihara, E.; He, N.; Hah, N.; Fan, W.; Pinto, A. F. M.; Huddy, T.; Wang, Y.; Ross, B.; Estepa, G.; Dai, Y.; Ding, N.; Sherman, M. H.; Fang, S.; Zhao, X.; Liddle, C.; Atkins, A. R.; Yu, R. T.; Downes, M.; Evans, R. M. Vitamin D switches BAF complexes to protect beta cells. Cell 2018, 173 (5), 1135-1149.

(34) Carlberg, C.; Campbell, M. J. Vitamin D receptor signaling mechanisms: Integrated actions of a well-defined transcription factor. Steroids 2013, 78 (2), 127-136.

(35) Carlberg, C.; Molnár, F. Current status of vitamin D signaling and its therapeutic applications. Curr. Top. Med. Chem. 2012, 12 (6), $528-547$.

(36) Pérez-García, X.; Rumbo, A.; Larriba, M. J.; Ordóñez, P.; Muñoz, A.; Mouriño, A. The first locked side-chain analogues of calcitriol $\left(1 \alpha, 25\right.$-dihydroxyvitamin $\left.\mathrm{D}_{3}\right)$ induce vitamin $\mathrm{D}$ receptor transcriptional activity. Org. Lett. 2003, 5, 4033-4036.

(37) Sigueiro, R.; Maestro, M. A.; Mouriño, A. Synthesis of sidechain locked analogs of $1 \alpha, 25$-dhydroxyvitamin $\mathrm{D}_{3}$ bearing a C17 methyl group. Org. Lett. 2018, 20 (9), 2641-2644.

(38) Norman, A. W.; Manchand, P. S.; Uskokovic, M. R.; Okamura, W. H.; Takeuchi, J. A.; Bishop, J. E.; Hisatake, J.-I.; Koeffler, H. P.; Peleg, S. Characterization of a novel analog of $1 \alpha, 25(\mathrm{OH})_{2}$-vitamin $\mathrm{D}_{3}$ with two side chains: interaction with its nuclear receptor and cellular actions. J. Med. Chem. 2000, 43, 2719-2730.

(39) Herdick, M.; Bury, Y.; Quack, M.; Uskokovic, M. R.; Polly, P.; Carlberg, C. Response element and coactivator-mediated conformational change of the vitamin $\mathrm{D}_{3}$ receptor permits sensitive interaction with agonists. Mol. Pharmacol. 2000, 57 (6), 1206-1217.

(40) Väisänen, S.; Peräkylä, M.; Kärkkäinen, J. I.; Uskokovic, M. R.; Carlberg, C. Structural evaluation of the agonistic action of a vitamin $\mathrm{D}$ analog with two side chains binding to the nuclear vitamin $\mathrm{D}$ receptor. Mol. Pharmacol. 2003, 63 (6), 1230-1237.

(41) Liu, Y. Y.; Collins, E. D.; Norman, A. W.; Peleg, S. Differential interaction of $1 \alpha, 25$-dihydroxyvitamin $\mathrm{D}_{3}$ analogues and their 20-epi homologues with the vitamin D receptor. J. Biol. Chem. 1997, 272 (6), 3336-3345.

(42) Maehr, H.; Lee, H. J.; Perry, B.; Suh, N.; Uskokovic, M. R. Calcitriol derivatives with two different side chains at C-20. V. Potent inhibitors of mammary carcinogenesis and inducers of leukemia differentiation. J. Med. Chem. 2009, 52 (17), 5505-5519.

(43) Okamoto, R.; Gery, S.; Kuwayama, Y.; Borregaard, N.; Ho, Q.; Alvarez, R.; Akagi, T.; Liu, G. Y.; Uskokovic, M. R.; Koeffler, H. P. Novel Gemini vitamin $\mathrm{D}_{3}$ analogs: large structure/function analysis and ability to induce antimicrobial peptide. Int. J. Cancer 2014, 134 (1), 207-217.

(44) Otero, R.; Seoane, S.; Sigueiro, R.; Belorusova, A. Y.; Maestro, M. A.; Perez-Fernandez, R.; Rochel, N.; Mouriño, A. Carborane-based 
design of a potent vitamin D receptor agonist. Chem. Sci. 2016, 7, 1033-1037.

(45) Takahashi, E.; Nakagawa, K.; Suhara, Y.; Kittaka, A.; Nihei, K.; Konno, K.; Takayama, H.; Ozono, K.; Okano, T. Biological activities of $2 \alpha$-substituted analogues of $1 \alpha, 25$-dihydroxyvitamin $\mathrm{D}_{3}$ in transcriptional regulation and human promyelocytic leukemia (HL60) cell proliferation and differentiation. Biol. Pharm. Bull. 2006, 29 (11), 2246-2250.

(46) Honzawa, S.; Takahashi, N.; Yamashita, A.; Sugiura, T.; Kurihara, M.; Arai, M. A.; Kato, S.; Kittaka, A. Synthesis of a $1 \alpha$-Cmethyl analogue of 25-hydroxyvitamin $\mathrm{D}_{3}$ : interaction with a mutant vitamin D receptor Arg274Leu. Tetrahedron 2009, 65 (34), 71357145.

(47) Sawada, D.; Tsukuda, Y.; Yasuda, K.; Sakaki, T.; Saito, H.; Takagi, K.; Takenouchi, K.; Chen, T. C.; Reddy, G. S.; Kittaka, A. Synthesis and biological activities of $1 \alpha, 4 \alpha, 25-$ and $1 \alpha, 4 \beta, 25-$ trihydroxyvitamin $\mathrm{D}_{3}$ and their metabolism by human CYP24A1 and UDP-glucuronosyltransferase. Chem. Pharm. Bull. 2012, 60 (10), $1343-1346$.

(48) Sibilska, I. K.; Szybinski, M.; Sicinski, R. R.; Plum, L. A.; Deluca, H. F. Highly potent 2-methylene analogs of $1 \alpha, 25$ dihydroxyvitamin $\mathrm{D}_{3}$ : synthesis and biological evaluation. J. Steroid Biochem. Mol. Biol. 2013, 136, 9-13.

(49) Piotrowska, A.; Wierzbicka, J.; Nadkarni, S.; Brown, G.; Kutner, A.; Zmijewski, M. A. Antiproliferative activity of double point modified analogs of 1,25-dihydroxyvitamin $\mathrm{D}_{2}$ against human malignant melanoma cell lines. Int. J. Mol. Sci. 2016, 17 (1), E76.

(50) Corcoran, A.; Nadkarni, S.; Yasuda, K.; Sakaki, T.; Brown, G.; Kutner, A.; Marcinkowska, E. Biological evaluation of double point modified analogues of 1,25-dihydroxyvitamin $\mathrm{D}_{2}$ as potential antileukemic agents. Int. J. Mol. Sci. 2016, 17 (2), E91.

(51) Saito, N.; Kittaka, A. Highly potent vitamin D receptor antagonists: design, synthesis, and biological evaluation. ChemBioChem 2006, 7 (10), 1478-1490.

(52) Saito, N.; Matsunaga, T.; Saito, H.; Anzai, M.; Takenouchi, K.; Miura, D.; Namekawa, J.; Ishizuka, S.; Kittaka, A. Further synthetic and biological studies on vitamin $\mathrm{D}$ hormone antagonists based on C24-alkylation and C2 $\alpha$-functionalization of 25-dehydro- $1 \alpha$-hydroxyvitamin $\mathrm{D}_{3}$-26,23-lactones. J. Med. Chem. 2006, 49 (24), 7063-7075.

(53) Sakamaki, Y.; Inaba, Y.; Yoshimoto, N.; Yamamoto, K. Potent antagonist for the vitamin $\mathrm{D}$ receptor: vitamin $\mathrm{D}$ analogues with simple side chain structure. J. Med. Chem. 2010, 53 (15), 5813-5826.

(54) Yoshimoto, N.; Sakamaki, Y.; Haeta, M.; Kato, A.; Inaba, Y.; Itoh, T.; Nakabayashi, M.; Ito, N.; Yamamoto, K. Butyl pocket formation in the vitamin $\mathrm{D}$ receptor strongly affects the agonistic or antagonistic behavior of ligands. J. Med. Chem. 2012, 55 (9), 43734381.

(55) Yoshino, M.; Eto, K.; Takahashi, K.; Ishihara, J.; Hatakeyama, S.; Ono, Y.; Saito, H.; Kubodera, N. Synthesis of 20-eldecalcitol [20epi-1 $\alpha, 25$-dihydroxy-2 $\beta$-(3-hydroxypropoxy)vitamin $\mathrm{D}_{3}$ : 20-epi-ED71]. Heterocycles 2010, 81, 381-394.

(56) Binderup, L.; Latini, S.; Binderup, E.; Bretting, C.; Calverley, M.; Hansen, K. 20-epi-vitamin $D_{3}$ analogues: a novel class of potent regulators of cell growth and immune responses. Biochem. Pharmacol. 1991, 42, 1569-1575.

(57) Paaren, H. F.; Schones, H. K.; DeLuca, H. F. Synthesis of $1 \beta$ hydroxyvitamin $\mathrm{D}_{3}$ and 1 1 ,25-dihydroxyvitamin $\mathrm{D}_{3}$. J. Chem. Soc., Chem. Commun. 1977, 890-892.

(58) Napoli, J. L.; Fivizzani, M. A.; Schnoes, H. K.; DeLuca, H. F. 1Fluorovitamin $\mathrm{D}_{3}$, a vitamin $\mathrm{D}_{3}$ analogue more active on bonecalcium mobilization than on intestinal-calcium transport. Biochemistry 1979, 18 (9), 1641-1646.

(59) Paaren, H. E.; Fivizzani, M. A.; Schnoes, H. K.; De Luca, H. F. 1 $\alpha, 25$-difluorovitamin $\mathrm{D}_{3}$ : an inert vitamin $\mathrm{D}$ analog. Arch. Biochem. Biophys. 1981, 209 (2), 579-583.

(60) Glebocka, A.; Chiellini, G. A-ring analogs of 1,25-dihydroxyvitamin $\mathrm{D}_{3}$. Arch. Biochem. Biophys. 2012, 523 (1), 48-57.
(61) Ishida, H.; Shimizu, M.; Yamamoto, K.; Iwasaki, Y.; Yamada, S.; Yamaguchi, K. Synthesis of 1-alkyl-1,25-dihydroxyvitamin $\mathrm{D}_{3}$. J. Org. Chem. 1995, 60, 1828-1833.

(62) Sekimoto, H.; Siu-Caldera, M. L.; Weiskopf, A.; Vouros, P.; Muralidharan, K. R.; Okamura, W. H.; Uskokovic, M. R.; Reddy, G. S. $1 \alpha, 25$-dihydroxy-3-epi-vitamin $\mathrm{D}_{3}$ : in vivo metabolite of $1 \alpha, 25$ dihydroxyvitamin $\mathrm{D}_{3}$ in rats. FEBS Lett. 1999, 448 (2-3), 278-282.

(63) Nakagawa, K.; Kurobe, M.; Ozono, K.; Konno, K.; Fujishima, T.; Takayama, H.; Okano, T. Novel ring A stereoisomers of 2-methyl$1 \alpha, 25$-dihydroxyvitamin $\mathrm{D}_{3}$ and 2-methyl-20-epi-1 $\alpha, 25$-dihydroxyvitamin $\mathrm{D}_{3}$ : transactivation of target genes and modulation of differentiation in human promyelocytic leukemia (HL-60) cells. Biochem. Pharmacol. 2000, 59 (6), 691-702.

(64) Suhara, Y.; Nihei, K. I.; Tanigawa, H.; Fujishima, T.; Konno, K.; Nakagawa, K.; Okano, T.; Takayama, H. Syntheses and biological evaluation of novel $2 \alpha$-substituted $1 \alpha, 25$-dihydroxyvitamin $\mathrm{D}_{3}$ analogues. Bioorg. Med. Chem. Lett. 2000, 10 (10), 1129-1132.

(65) Perlman, K. L.; Swenson, R. E.; Paaren, H. E.; Schnoes, H. K.; DeLuca, H. F. Novel synthesis of 19-nor-vitamin D compounds. Tetrahedron Lett. 1991, 32, 7663-7666.

(66) Bouillon, R.; Sarandeses, L. A.; Allewaert, K.; Zhao, J.; Mascarenas, J. L.; Mouriño, A.; Vrielynck, S.; de Clercq, P.; Vandewalle, M. Biologic activity of dihydroxylated 19-nor-(pre)vitamin $\mathrm{D}_{3}$. J. Bone Miner. Res. 1993, 8 (8), 1009-1015.

(67) Sicinski, R. R.; Perlman, K. L.; DeLuca, H. F. Synthesis and biological activity of 2-hydroxy and 2-alkoxy analogs of 1 $\alpha, 25$ dihydroxy-19-norvitamin $\mathrm{D}_{3}$. J. Med. Chem. 1994, 37 (22), 37303738.

(68) Yamamoto, H.; Shevde, N. K.; Warrier, A.; Plum, L. A.; DeLuca, H. F.; Pike, J. W. 2-Methylene-19-nor-(20S)-1,25-dihydroxyvitamin $\mathrm{D}_{3}$ potently stimulates gene-specific DNA binding of the vitamin D receptor in osteoblasts. J. Biol. Chem. 2003, 278 (34), 31756-31765.

(69) Williams, S.; Bledsoe, R. K.; Collins, J. L.; Boggs, S.; Lambert, M. H.; Miller, A. B.; Moore, J.; McKee, D. D.; Moore, L.; Nichols, J.; Parks, D.; Watson, M.; Wisely, B.; Willson, T. M. X-ray crystal structure of the liver $\mathrm{X}$ receptor beta ligand binding domain: regulation by a histidine-tryptophan switch. J. Biol. Chem. 2003, 278 (29), 27138-27143.

(70) Tsugawa, N.; Nakagawa, K.; Kurobe, M.; Ono, Y.; Kubodera, N.; Ozono, K.; Okano, T. In vitro biological activities of a series of 2 beta-substituted analogues of $1 \alpha, 25$-dihydroxyvitamin $\mathrm{D}_{3}$. Biol. Pharm. Bull. 2000, 23 (1), 66-71.

(71) Shimizu, M.; Miyamoto, Y.; Kobayashi, E.; Shimazaki, M.; Yamamoto, K.; Reischl, W.; Yamada, S. Synthesis and biological activities of new 1 $\alpha, 25$-dihydroxy-19norvitamin $\mathrm{D}_{3}$ analogs with modifications in both A-ring and the side chain. Bioorg. Med. Chem. 2006, 14, 4277-4294.

(72) Posner, G. H.; Lee, J. K.; Wang, Q.; Peleg, S.; Burke, M.; Brem, H.; Dolan, P.; Kensler, T. W. Noncalcemic, antiproliferative, transcriptionally active, 24-fluorinated hybrid analogues of the hormone $1 \alpha, 25$-dihydroxyvitamin $\mathrm{D}_{3}$ : synthesis and preliminary biological evaluation. J. Med. Chem. 1998, 41, 3008-3014.

(73) Peleg, S.; Ismail, A.; Uskokovic, M. R.; Avnur, Z. Evidence for tissue- and cell-type selective activation of the vitamin $\mathrm{D}$ receptor by Ro-26-9228, a noncalcemic analog of vitamin $\mathrm{D}_{3}$. J. Cell. Biochem. 2003, 88 (2), 267-273.

(74) Flores, A.; Sicinski, R. R.; Grzywacz, P.; Thoden, J. B.; Plum, L. A.; Clagett-Dame, M.; DeLuca, H. F. A $20 \mathrm{~S}$ combined with a $22 \mathrm{R}$ configuration markedly increases both in vivo and in vitro biological activity of 1 $\alpha, 25$-dihydroxy-22-methyl-2-methylene-19-norvitamin $\mathrm{D}_{3}$. J. Med. Chem. 2012, 55 (9), 4352-4366.

(75) Sibilska, I. K.; Szybinski, M.; Sicinski, R. R.; Plum, L. A.; DeLuca, H. F. Synthesis and biological activity of 2-methylene analogues of calcitriol and related compounds. J. Med. Chem. 2015, 58 (24), 9653-9662.

(76) Sawada, D.; Tsukuda, Y.; Saito, H.; Kakuda, S.; TakimotoKamimura, M.; Ochiai, E.; Takenouchi, K.; Kittaka, A. Development of 14-epi-19-nortachysterol and its unprecedented binding config- 
uration for the human vitamin D receptor. J. Am. Chem. Soc. 2011, 133, 7215-7221.

(77) Laverny, G.; Penna, G.; Uskokovic, M.; Marczak, S.; Maehr, H.; Jankowski, P.; Ceailles, C.; Vouros, P.; Smith, B.; Robinson, M.; Reddy, G. S.; Adorini, L. Synthesis and anti-inflammatory properties of 1 $\alpha, 25$-dihydroxy-16-ene-20-cyclopropyl-24-oxo-vitamin $\mathrm{D}_{3}$, a hypocalcemic, stable metabolite of $1 \alpha, 25$-dihydroxy-16-ene-20-cyclopropyl-vitamin $\mathrm{D}_{3}$. J. Med. Chem. 2009, 52 (8), 2204-2213.

(78) Fujii, S.; Masuno, H.; Taoda, Y.; Kano, A.; Wongmayura, A.; Nakabayashi, M.; Ito, N.; Shimizu, M.; Kawachi, E.; Hirano, T.; Endo, Y.; Tanatani, A.; Kagechika, H. Boron cluster-based development of potent nonsecosteroidal vitamin $\mathrm{D}$ receptor ligands: direct observation of hydrophobic interaction between protein surface and carborane. J. Am. Chem. Soc. 2011, 133 (51), 20933-20941.

(79) Boehm, M. F.; Fitzgerald, P.; Zou, A.; Elgort, M. G.; Bischoff, E. D.; Mere, L.; Mais, D. E.; Bissonnette, R. P.; Heyman, R. A.; Nadzan, A. M.; Reichman, M.; Allegretto, E. A. Novel nonsecosteroidal vitamin $\mathrm{D}$ mimics exert VDR-modulating activities with less calcium mobilization than 1,25-dihydroxyvitamin $\mathrm{D}_{3}$. Chem. Biol. 1999, 6 (5), 265-275.

(80) Kashiwagi, H.; Ohta, M.; Ono, Y.; Morikami, K.; Itoh, S.; Sato, H.; Takahashi, T. Effects of fluorines on nonsecosteroidal vitamin D receptor agonists. Bioorg. Med. Chem. 2013, 21 (3), 712-721.

(81) Hao, M.; Hou, S.; Xue, L.; Yuan, H.; Zhu, L.; Wang, C.; Wang, B.; Tang, C.; Zhang, C. Further developments of the phenyl-pyrrolyl pentane series of nonsteroidal vitamin $\mathrm{D}$ receptor modulators as anticancer agents. J. Med. Chem. 2018, 61 (7), 3059-3075.

(82) Shen, W.; Xue, J.; Zhao, Z.; Zhang, C. Novel nonsecosteroidal VDR agonists with phenyl-pyrrolyl pentane skeleton. Eur. J. Med. Chem. 2013, 69, 768-778.

(83) Gogoi, P.; Seoane, S.; Sigueiro, R.; Guiberteau, T.; Maestro, M. A.; Perez-Fernandez, R.; Rochel, N.; Mouriño, A. Aromatic-based design of highly active and noncalcemic vitamin $\mathrm{D}$ receptor agonists. J. Med. Chem. 2018, 61 (11), 4928-4937.

(84) Gogoi, P.; Sigueiro, R.; Eduardo, S.; Mouriño, A. An expeditious route to $1 \alpha, 25$-dihydroxyvitamin $\mathrm{D}_{3}$ and its analogues by an aqueous tandem palladium-catalyzed A-ring closure and suzuki coupling to the C/D unit. Chem. - Eur. J. 2010, 16 (5), 1432-1435.

(85) Stio, M.; Martinesi, M.; Bruni, S.; Treves, C.; Mathieu, C.; Verstuyf, A.; d'Albasio, G.; Bagnoli, S.; Bonanomi, A. G. The vitamin $\mathrm{D}$ analogue TX 527 blocks NF-kappaB activation in peripheral blood mononuclear cells of patients with Crohn's disease. J. Steroid Biochem. Mol. Biol. 2007, 103 (1), 51-60.

(86) Sherman, M. H.; Yu, R. T.; Engle, D. D.; Ding, N.; Atkins, A. R.; Tiriac, H.; Collisson, E. A.; Connor, F.; Van Dyke, T.; Kozlov, S.; Martin, P.; Tseng, T. W.; Dawson, D. W.; Donahue, T. R.; Masamune, A.; Shimosegawa, T.; Apte, M. V.; Wilson, J. S.; Ng, B.; Lau, S. L.; Gunton, J. E.; Wahl, G. M.; Hunter, T.; Drebin, J. A.; O’Dwyer, P. J.; Liddle, C.; Tuveson, D. A.; Downes, M.; Evans, R. M. Vitamin D receptor-mediated stromal reprogramming suppresses pancreatitis and enhances pancreatic cancer therapy. Cell 2014, 159 (1), 80-93.

(87) Carlberg, C. Genome-wide (over)view on the actions of vitamin D. Front. Physiol. 2014, 5, 167.

(88) Plum, L. A.; DeLuca, H. F. Vitamin D, disease and therapeutic opportunities. Nat. Rev. Drug Discovery 2010, 9 (12), 941-955.

(89) Leyssens, C.; Verlinden, L.; Verstuyf, A. The future of vitamin D analogs. Front. Physiol. 2014, 5, 122.

(90) Tocchini-Valentini, G.; Rochel, N.; Wurtz, J. M.; Mitschler, A.; Moras, D. Crystal structures of the vitamin D receptor complexed to superagonist 20-epi ligands. Proc. Natl. Acad. Sci. U. S. A. 2001, 98 (10), 5491-5496.

(91) Tocchini-Valentini, G.; Rochel, N.; Wurtz, J. M.; Moras, D. Crystal structures of the vitamin D nuclear receptor liganded with the vitamin D side chain analogues calcipotriol and seocalcitol, receptor agonists of clinical importance: insights into a structural basis for the switching of calcipotriol to a receptor antagonist by further side chain modification. J. Med. Chem. 2004, 47 (8), 1956-1961.
(92) Eelen, G.; Verlinden, L.; Rochel, N.; Claessens, F.; De Clercq, P.; Vandewalle, M.; Tocchini-Valentini, G.; Moras, D.; Bouillon, R.; Verstuyf, A. Superagonistic action of 14-epi-analogs of 1,25dihydroxyvitamin D explained by vitamin $\mathrm{D}$ receptor-coactivator interaction. Mol. Pharmacol. 2005, 67 (5), 1566-1573.

(93) Hourai, S.; Fujishima, T.; Kittaka, A.; Suhara, Y.; Takayama, H.; Rochel, N.; Moras, D. Probing a water channel near the A-ring of receptor-bound $1 \alpha, 25$-dihydroxyvitamin $\mathrm{D}_{3}$ with selected $2 \alpha$-substituted analogues. J. Med. Chem. 2006, 49, 5199-5205.

(94) Kakuda, S.; Ishizuka, S.; Eguchi, H.; Mizwicki, M. T.; Norman, A. W.; Takimoto-Kamimura, M. Structural basis of the histidinemediated vitamin $\mathrm{D}$ receptor agonistic and antagonistic mechanisms of (23S)-25-dehydro-1 $\alpha$-hydroxyvitamin $\mathrm{D}_{3}$-26,23-lactone. Acta Crystallogr., Sect. D: Biol. Crystallogr. 2010, 66 (Part 8), 918-926.

(95) Antony, P.; Sigüeiro, R.; Huet, T.; Sato, Y.; Ramalanjaona, N.; Rodrigues, L. C.; Mouriño, A.; Moras, D.; Rochel, N. Structurefunction relationships and crystal structures of the vitamin $\mathrm{D}$ receptor bound $2 \alpha$-methyl-(20S,23S)- and $2 \alpha$-methyl-(20S,23R)-epoxymethano-1 $\alpha, 25$-dihydroxyvitamin $\mathrm{D}_{3}$. J. Med. Chem. 2010, 53 (3), 11591171.

(96) Molnár, F.; Sigüeiro, R.; Sato, Y.; Araujo, C.; Schuster, I.; Antony, P.; Peluso, J.; Muller, C.; Mouriño, A.; Moras, D.; Rochel, N. $1 \alpha, 25(\mathrm{OH})_{2}$-3-epi-vitamin $\mathrm{D}_{3}$, a natural physiological metabolite of vitamin $\mathrm{D}_{3}$ : its synthesis, biological activity and crystal structure with its receptor. PLoS One 2011, 6, e18124.

(97) Shindo, K.; Kumagai, G.; Takano, M.; Sawada, D.; Saito, N.; Saito, H.; Kakuda, S.; Takagi, K.; Ochiai, E.; Horie, K.; TakimotoKamimura, M.; Ishizuka, S.; Takenouchi, K.; Kittaka, A. New C15substituted active vitamin $\mathrm{D}_{3}$. Org. Lett. 2011, 13, 2852-2855.

(98) Kashiwagi, H.; Ono, Y.; Shimizu, K.; Haneishi, T.; Ito, S.; Iijima, S.; Kobayashi, T.; Ichikawa, F.; Harada, S.; Sato, H.; Sekiguchi, N.; Ishigai, M.; Takahashi, T. Novel nonsecosteroidal vitamin $\mathrm{D}_{3}$ carboxylic acid analogs for osteoporosis, and SAR analysis. Bioorg. Med. Chem. 2011, 19 (16), 4721-4729.

(99) Hourai, S.; Rodrigues, L.; Antony, P.; Reina-San-Martin, B.; Ciesielski, F.; Magnier, B.; Schoonjans, K.; Mouriño, A.; Rochel, N.; Moras, D. Structure-based design of a superagonist ligand for the vitamin D nuclear receptor. Chem. Biol. 2008, 15, 383-392.

(100) Rochel, N.; Moras, D. Crystal structure of a vitamin $\mathrm{D}_{3}$ analog, ZK203278, showing dissociated profile. Anticancer Res. 2012, 32 (1), 335-339.

(101) Rochel, N.; Hourai, S.; Moras, D. Crystal structure of hereditary vitamin D-resistant rickets-associated mutant H305Q of vitamin D nuclear receptor bound to its natural ligand. J. Steroid Biochem. Mol. Biol. 2010, 121, 84-87.

(102) Verlinden, L.; Verstuyf, A.; Eelen, G.; Bouillon, R.; OrdóñezMorán, P.; Larriba, M. J.; Muñoz, A.; Rochel, N.; Sato, Y.; Moras, D.; Maestro, M.; Seoane, S.; Dominguez, F.; Eduardo-Canosa, S.; Nicoletti, D.; Moman, E.; Mouriño, A. Synthesis, structure, and biological activity of des-side chain analogues of $1 \alpha, 25$-dihydroxyvitamin $\mathrm{D}_{3}$ with substituents at $\mathrm{C} 18$. ChemMedChem 2011, 6 (5), 788-793.

(103) Fraga, R.; Zacconi, F.; Sussman, F.; Ordonez-Moran, P.; Muñoz, A.; Huet, T.; Molnár, F.; Moras, D.; Rochel, N.; Maestro, M.; Mouriño, A. Design, synthesis, evaluation, and structure of vitamin D analogues with furan side chains. Chem. - Eur. J. 2012, 18 (2), 603612.

(104) Saitoh, H.; Chida, T.; Takagi, K.; Horie, K.; Sawai, Y.; Nakamura, Y.; Harada, Y.; Takenouchi, K.; Kittaka, A. Synthesis of C2 substituted vitamin $\mathrm{D}$ derivatives having ringed side chains and their biological evaluation, especially biological effect on bone by modification at the C-2 position. Org. Biomol. Chem. 2011, 9 (10), 3954-3964.

(105) Kashiwagi, H.; Ono, Y.; Ohta, M.; Itoh, S.; Ichikawa, F.; Harada, S.; Takeda, S.; Sekiguchi, N.; Ishigai, M.; Takahashi, T. A series of nonsecosteroidal vitamin $\mathrm{D}$ receptor agonists for osteoporosis therapy. Bioorg. Med. Chem. 2013, 21 (7), 1823-1833.

(106) Saitoh, H.; Watanabe, H.; Kakuda, S.; Takimoto-Kamimura, M.; Takagi, K.; Takeuchi, A.; Takenouchi, K. Synthesis and biological 
activities of vitamin $\mathrm{D}_{3}$ derivatives with cyanoalkyl side chain at $\mathrm{C}-2$ position. J. Steroid Biochem. Mol. Biol. 2015, 148, 27-30.

(107) Sawada, D.; Kakuda, S.; Kamimura-Takimoto, M.; Takeuchi, A.; Matsumoto, Y.; Kittaka, A. Revisiting the 7,8-cis-vitamin $\mathrm{D}_{3}$ derivatives: Synthesis, evaluating the biological activity, and study of the binding configuration. Tetrahedron 2016, 72, 2838-2848.

(108) Ciesielski, F.; Sato, Y.; Chebaro, Y.; Moras, D.; Dejaegere, A.; Rochel, N. Structural basis for the accommodation of bis- and trisaromatic derivatives in vitamin $\mathrm{D}$ nuclear receptor. J. Med. Chem. 2012, 55 (19), 8440-8449.

(109) Matsuo, M.; Hasegawa, A.; Takano, M.; Saito, H.; Kakuda, S.; Chida, T.; Takagi, K.; Ochiai, E.; Horie, K.; Harada, Y.; TakimotoKamimura, M.; Takenouchi, K.; Sawada, D.; Kittaka, A. Synthesis of $2 \alpha$-heteroarylalkyl active vitamin $\mathrm{D}_{3}$ with therapeutic effect on enhancing bone mineral density in vivo. ACS Med. Chem. Lett. 2013, 4 (7), 671-674.

(110) Belorusova, A. Y.; Eberhardt, J.; Potier, N.; Stote, R. H.; Dejaegere, A.; Rochel, N. Structural insights into the molecular mechanism of vitamin $\mathrm{D}$ receptor activation by lithocholic acid involving a new mode of ligand recognition. J. Med. Chem. 2014, 57 (11), 4710-4719.

(111) Zheng, J.; Chang, M. R.; Stites, R. E.; Wang, Y.; Bruning, J. B.; Pascal, B. D.; Novick, S. J.; Garcia-Ordonez, R. D.; Stayrook, K. R.; Chalmers, M. J.; Dodge, J. A.; Griffin, P. R. HDX reveals the conformational dynamics of DNA sequence specific VDR co-activator interactions. Nat. Commun. 2017, 8 (1), 923.

(112) Sawada, D.; Kakuda, S.; Takeuchi, A.; Kawagoe, F.; TakimotoKamimura, M.; Kittaka, A. Effects of 2-substitution on 14-epi-19nortachysterol-mediated biological events: based on synthesis and Xray co-crystallographic analysis with the human vitamin $\mathrm{D}$ receptor. Org. Biomol. Chem. 2018, 16 (14), 2448-2455.

(113) Vanhooke, J. L.; Benning, M. M.; Bauer, C. B.; Pike, J. W.; DeLuca, H. F. Molecular structure of the rat vitamin D receptor ligand binding domain complexed with 2-carbon-substituted vitamin $\mathrm{D}_{3}$ hormone analogues and a LXXLL-containing coactivator peptide. Biochemistry 2004, 43 (14), 4101-4110.

(114) Vanhooke, J.; Tadi, B.; Benning, M.; Plum, L.; DeLuca, H. New analogs of 2-methylene-19-nor-(20S)-1,25-dihydroxyvitamin $\mathrm{D}_{3}$ with conformationally restricted side chains: evaluation of biological activity and structural determination of VDR-bound conformations. Arch. Biochem. Biophys. 2007, 460, 161-165.

(115) Kakuda, S.; Okada, K.; Eguchi, H.; Takenouchi, K.; Hakamata, W.; Kurihara, M.; Takimoto-Kamimura, M. Structure of the ligandbinding domain of rat VDR in complex with the nonsecosteroidal vitamin $\mathrm{D}_{3}$ analogue YR301. Acta Crystallogr., Sect. F: Struct. Biol. Cryst. Commun. 2008, 64, 970-973.

(116) Shimizu, M.; Miyamoto, Y.; Takaku, H.; Matsuo, M.; Nakabayashi, M.; Masuno, H.; Udagawa, N.; DeLuca, H.; Ikura, T.; Ito, N. 2-Substituted-16-ene-22-thia-1 $\alpha, 25$-dihydroxy-26,27-dimethyl19-norvitamin $\mathrm{D}_{3}$ analogs: Synthesis, biological evaluation, and crystal structure. Bioorg. Med. Chem. 2008, 16, 6949-6964.

(117) Nakabayashi, M.; Yamada, S.; Yoshimoto, N.; Tanaka, T.; Igarashi, M.; Ikura, T.; Ito, N.; Makishima, M.; Tokiwa, H.; DeLuca, H.; Shimizu, M. Crystal structures of rat vitamin D receptor bound to adamantyl vitamin D analogs: structural basis for vitamin D receptor antagonism and partial agonism. J. Med. Chem. 2008, 51, 5320-5329.

(118) Inaba, Y.; Yoshimoto, N.; Sakamaki, Y.; Nakabayashi, M.; Ikura, T.; Tamamura, H.; Ito, N.; Shimizu, M.; Yamamoto, K. A new class of vitamin $\mathrm{D}$ analogues that induce structural rearrangement of the ligand-binding pocket of the receptor. J. Med. Chem. 2009, 52, 1438-1449.

(119) Inaba, Y.; Nakabayashi, M.; Itoh, T.; Yoshimoto, N.; Ikura, T.; Ito, N.; Shimizu, M.; Yamamoto, K. 22S-butyl-1 $\alpha, 24 \mathrm{R}$-dihydroxyvitamin $\mathrm{D}_{3}$ : Recovery of vitamin $\mathrm{D}$ receptor agonistic activity. J. Steroid Biochem. Mol. Biol. 2010, 121 (1-2), 146-150.

(120) Demizu, Y.; Takahashi, T.; Kaneko, F.; Sato, Y.; Okuda, H.; Ochiai, E.; Horie, K.; Takagi, K.; Kakuda, S.; Takimoto-Kamimura, M.; Kurihara, M. Design, synthesis and X-ray crystallographic study of new nonsecosteroidal vitamin D receptor ligands. Bioorg. Med. Chem. Lett. 2011, 21 (20), 6104-6107.

(121) Nakabayashi, M.; Tsukahara, Y.; Iwasaki-Miyamoto, Y.; Mihori-Shimazaki, M.; Yamada, S.; Inaba, S.; Oda, M.; Shimizu, M.; Makishima, M.; Tokiwa, H.; Ikura, T.; Ito, N. Crystal structures of hereditary vitamin $\mathrm{D}$-resistant rickets-associated vitamin $\mathrm{D}$ receptor mutants R270L and W282R bound to 1,25-dihydroxyvitamin $\mathrm{D}_{3}$ and synthetic ligands. J. Med. Chem. 2013, 56 (17), 6745-6760.

(122) Kudo, T.; Ishizawa, M.; Maekawa, K.; Nakabayashi, M.; Watarai, Y.; Uchida, H.; Tokiwa, H.; Ikura, T.; Ito, N.; Makishima, M.; Yamada, S. Combination of triple bond and adamantane ring on the vitamin $\mathrm{D}$ side chain produced partial agonists for vitamin D receptor. J. Med. Chem. 2014, 57 (10), 4073-4087.

(123) Asano, L.; Ito, I.; Kuwabara, N.; Waku, T.; Yanagisawa, J.; Miyachi, H.; Shimizu, T. Structural basis for vitamin D receptor agonism by novel non-secosteroidal ligands. FEBS Lett. 2013, 587, 957-963.

(124) Masuno, H.; Ikura, T.; Morizono, D.; Orita, I.; Yamada, S.; Shimizu, M.; Ito, N. Crystal structures of complexes of vitamin D receptor ligand-binding domain with lithocholic acid derivatives. $J$. Lipid Res. 2013, 54 (8), 2206-2213.

(125) Anami, Y.; Itoh, T.; Egawa, D.; Yoshimoto, N.; Yamamoto, K. A mixed population of antagonist and agonist binding conformers in a single crystal explains partial agonism against vitamin $\mathrm{D}$ receptor: active vitamin D analogues with 22R-alkyl group. J. Med. Chem. 2014, 57 (10), 4351-4367.

(126) Watarai, Y.; Ishizawa, M.; Ikura, T.; Zacconi, F. C.; Uno, S.; Ito, N.; Mouriño, A.; Tokiwa, H.; Makishima, M.; Yamada, S. Synthesis, biological activities, and X-ray crystal structural analysis of 25-hydroxy-25(or 26)-adamantyl-17-[20(22),23-diynyl]-21-norvitamin D compounds. J. Med. Chem. 2015, 58 (24), 9510-9521.

(127) Anami, Y.; Sakamaki, Y.; Itoh, T.; Inaba, Y.; Nakabayashi, M.; Ikura, T.; Ito, N.; Yamamoto, K. Fine tuning of agonistic/antagonistic activity for vitamin $\mathrm{D}$ receptor by 22 -alkyl chain length of ligands: 22S-Hexyl compound unexpectedly restored agonistic activity. Bioorg. Med. Chem. 2015, 23 (22), 7274-7281.

(128) Anami, Y.; Shimizu, N.; Ekimoto, T.; Egawa, D.; Itoh, T.; Ikeguchi, M.; Yamamoto, K. Apo- and antagonist-binding structures of vitamin $\mathrm{D}$ receptor ligand-binding domain revealed by hybrid approach combining small-angle X-ray scattering and molecular dynamics. J. Med. Chem. 2016, 59 (17), 7888-7900.

(129) Kato, A.; Itoh, T.; Anami, Y.; Egawa, D.; Yamamoto, K. Helix12-stabilization antagonist of vitamin $\mathrm{D}$ receptor. Bioconjugate Chem. 2016, 27 (7), 1750-1761.

(130) Asano, L.; Waku, T.; Abe, R.; Kuwabara, N.; Ito, I.; Yanagisawa, J.; Nagasawa, K.; Shimizu, T. Regulation of the vitamin D receptor by vitamin D lactam derivatives. FEBS Lett. 2016, 590 (18), 3270-3279.

(131) Egawa, D.; Itoh, T.; Kato, A.; Kataoka, S.; Anami, Y.; Yamamoto, K. SRC2-3 binds to vitamin D receptor with high sensitivity and strong affinity. Bioorg. Med. Chem. 2017, 25 (2), 568574 .

(132) Kato, A.; Yamao, M.; Hashihara, Y.; Ishida, H.; Itoh, T.; Yamamoto, K. Vitamin D analogues with a p-hydroxyphenyl group at the $\mathrm{C} 25$ position: Crystal structure of vitamin D receptor ligandbinding domain complexed with the ligand explains the mechanism underlying full antagonistic action. J. Med. Chem. 2017, 60 (20), $8394-8406$

(133) Otero, R.; Ishizawa, M.; Numoto, N.; Ikura, T.; Ito, N.; Tokiwa, H.; Mouriño, A.; Makishima, M.; Yamada, S. 25 SAdamantyl-23-yne-26,27-dinor-1 $\alpha, 25$-dihydroxyvitamin $\mathrm{D}_{3}$ : synthesis, tissue selective bological activities, and X-ray crystal structural analysis of its vitamin D receptor complex. J. Med. Chem. 2018, 61 (15), $6658-6673$.

(134) Yoshizawa, M.; Itoh, T.; Hori, T.; Kato, A.; Anami, Y.; Yoshimoto, N.; Yamamoto, K. Identification of the histidine residue in vitamin $\mathrm{D}$ receptor that covalently binds to electrophilic ligands. $J$. Med. Chem. 2018, 61 (14), 6339-6349. 
(135) Rochel, N.; Hourai, S.; Perez-Garcia, X.; Rumbo, A.; Mouriño, A.; Moras, D. Crystal structure of the vitamin D nuclear receptor ligand binding domain in complex with a locked side chain analog of calcitriol. Arch. Biochem. Biophys. 2007, 460 (2), 172-176.

(136) Ciesielski, F.; Rochel, N.; Moras, D. Adaptability of the vitamin $\mathrm{D}$ nuclear receptor to the synthetic ligand Gemini: remodelling the LBP with one side chain rotation. J. Steroid Biochem. Mol. Biol. 2007, 103, 235-242.

(137) Eelen, G.; Valle, N.; Sato, Y.; Rochel, N.; Verlinden, L.; De Clercq, P.; Moras, D.; Bouillon, R.; Muñoz, A.; Verstuyf, A. Superagonistic fluorinated vitamin $\mathrm{D}_{3}$ analogs stabilize helix 12 of the vitamin D receptor. Chem. Biol. 2008, 15 (10), 1029-1034.

(138) Huet, T.; Maehr, H.; Lee, H.; Uskokovic, M.; Suh, N.; Moras, D.; Rochel, N. Structure function study of Gemini derivatives with two different side chains at C-20, Gemini-0072 and Gemini-0097. MedChem Comm 2011, 2, 424-429.

(139) Fischer, J.; Wang, T. T.; Kaldre, D.; Rochel, N.; Moras, D.; White, J. H.; Gleason, J. L. Synthetically accessible non-secosteroidal hybrid molecules combining vitamin $\mathrm{D}$ receptor agonism and histone deacetylase inhibition. Chem. Biol. 2012, 19 (8), 963-971.

(140) Huet, T.; Laverny, G.; Ciesielski, F.; Molnar, F.; Ramamoorthy, T. G.; Belorusova, A. Y.; Antony, P.; Potier, N.; Metzger, D.; Moras, D.; Rochel, N. A vitamin D receptor selectively activated by Gemini analogs reveals ligand dependent and independent effects. Cell Rep. 2015, 10 (4), 516-526.

(141) Belorusova, A. Y.; Suh, N.; Lee, H. J.; So, J. Y.; Maehr, H.; Rochel, N. Structural analysis and biological activities of BXL0124, a Gemini analog of vitamin D. J. Steroid Biochem. Mol. Biol. 2017, 173, $69-74$.

(142) Lin, Z.; Chen, H.; Belorusova, A. Y.; Bollinger, J. C.; Tang, E. K. Y.; Janjetovic, Z.; Kim, T. K.; Wu, Z.; Miller, D. D.; Slominski, A. T.; Postlethwaite, A. E.; Tuckey, R. C.; Rochel, N.; Li, W. 1 $\alpha$,20SDihydroxyvitamin $\mathrm{D}_{3}$ interacts with vitamin $\mathrm{D}$ receptor: Crystal structure and route of chemical synthesis. Sci. Rep. 2017, 7 (1), 10193. (143) Belorusova, A. Y.; Martinez, A.; Gandara, Z.; Gomez, G.; Fall, Y.; Rochel, N. Structure-activity relationship study of vitamin D analogs with oxolane group in their side chain. Eur. J. Med. Chem. 2017, 134, 86-96.

(144) Lin, Z.; Marepally, S. R.; Goh, E. S. Y.; Cheng, C. Y. S.; Janjetovic, Z.; Kim, T. K.; Miller, D. D.; Postlethwaite, A. E.; Slominski, A. T.; Tuckey, R. C.; Peluso-Iltis, C.; Rochel, N.; Li, W. Investigation of $20 \mathrm{~S}$-hydroxyvitamin $\mathrm{D}_{3}$ analogs and their $1 \alpha-\mathrm{OH}$ derivatives as potent vitamin $\mathrm{D}$ receptor agonists with antiinflammatory activities. Sci. Rep. 2018, 8 (1), 1478. 